\title{
HDAC6-mediated acetylation of lipid droplet-binding protein CIDEC regulates fat-induced lipid storage
}

\begin{abstract}
Hui Qian, ${ }^{1}$ Yuanying Chen, ${ }^{1}$ Zongqian Nian, ${ }^{1}$ Lu Su, ${ }^{1}$ Haoyong Yu, ${ }^{2}$ Feng-Jung Chen, ${ }^{1}$ Xiuqin Zhang, ${ }^{3}$ Wenyi Xu, ${ }^{1}$ Linkang Zhou, ${ }^{1}$ Jiaming Liu, ${ }^{1}$ Jinhai Yu, ${ }^{1}$ Luxin Yu, ${ }^{1}$ Yan Gao, ${ }^{4}$ Hongchao Zhang, ${ }^{5}$ Haihong Zhang, ${ }^{6}$ Shimin Zhao, ${ }^{7}$ Li Yu, ${ }^{8}$ Rui-Ping Xiao, ${ }^{9}$ Yuqian Bao, ${ }^{2}$ Shaocong Hou, ${ }^{10}$ Pingping Li, ${ }^{10}$ Jiada Li, ${ }^{11}$ Haiteng Deng, ${ }^{4}$ Weiping Jia, ${ }^{2}$ and Peng Li ${ }^{1}$

1MOE Key Laboratory of Bioinformatics and Tsinghua-Peking Center for Life Sciences, Beijing Advanced Innovation Center for Structural Biology, School of Life Sciences, Tsinghua University, Beijing, China. ${ }^{2}$ Shanghai Key Laboratory of Diabetes Mellitus, Department of Endocrinology and Metabolism, Shanghai JiaoTong University Affiliated Sixth People's Hospital, Shanghai Diabetes Institute, Shanghai Clinical Center for Diabetes, and Shanghai Key Clinical Center for Metabolic Disease, Shanghai, China. ${ }^{3}$ Institute of Molecular Medicine, Beijing Key Laboratory of Cardiometabolic Molecular Medicine, Peking University, Beijing, China. ${ }^{4}$ Proteomics Facility, School of Life Sciences, Tsinghua University, Beijing, China. ${ }^{5}$ Heart Center and ${ }^{6}$ Department of General Surgery, Air Force General Hospital, PLA, Beijing, China. ${ }^{7}$ State Key Laboratory of Genetic Engineering, School of Life Sciences, Institutes of Biomedical Sciences, Fudan University, Shanghai, China. ${ }^{8}$ State Key Laboratory of Biomembrane and Membrane Biotechnology, Tsinghua University-Peking University Joint Center for Life Sciences, School of Life Sciences, Tsinghua University, Beijing, China. ${ }^{9}$ Institute of Molecular Medicine, State Key Laboratory of Biomembrane and Membrane Biotechnology, Peking-Tsinghua Center for Life Sciences, Beijing Key Laboratory of Cardiometabolic Molecular Medicine, Peking University, Beijing, China. ${ }^{10}$ State Key Laboratory of Bioactive Substance and Function of Natural Medicines, Institute of Materia Medica, Chinese Academy of Medical Sciences and Peking Union Medical College, Diabetes Research Center of Chinese Academy of Medical Sciences, Beijing, China. "'State Key Laboratory of Medical Genetics and School of Life Sciences, Central South University, Changsha, China.
\end{abstract}

\begin{abstract}
Obesity is characterized by aberrant fat accumulation. However, the intracellular signaling pathway that senses dietary fat and leads to fat storage remains elusive. Here, we have observed that the levels of histone deacetylase 6 (HDAC6) and the related family member HDAC10 are markedly reduced in adipose tissues of obese animals and humans. Mice with adipocyte-specific depletion of Hdac6 exhibited increased fat accumulation and reduced insulin sensitivity. In normal adipocytes, we found that reversal of P300/CBP-associated factor-induced (PCAF-induced) acetylation at K56 on cell death-inducing DFFA-like effector C (CIDEC, also known as FSP27) critically regulated lipid droplet fusion and lipid storage. Importantly, HDAC6 deacetylates CIDEC, leading to destabilization and reduced lipid droplet fusion. Accordingly, we observed elevated levels of CIDEC and its acetylated form in HDAC-deficient adipocytes as well as the adipose tissue of obese animals and humans. Fatty acids (FAs) prevented CIDEC deacetylation by promoting the dissociation of CIDEC from HDAC6, which resulted in increased association of CIDEC with PCAF on the endoplasmic reticulum. Control of CIDEC acetylation required the conversion of FAs to triacylglycerols. Thus, we have revealed a signaling axis that is involved in the coordination of nutrient availability, protein acetylation, and cellular lipid metabolic responses.
\end{abstract}

\section{Introduction}

Levels of protein acetylation have been shown to affect the activity of many proteins, and its aberrant regulation is linked to the development of cancer and metabolic diseases (1-4). Protein acetylation is modulated by the antagonistic actions of histone acetyltransferases (HATs) and histone deacetylases (HDACs) (5, 6). HDAC6, a member of the class IIb HDACs that contains two deacetylase domains and a ubiquitin-binding domain at its C-terminus, is the major cytosolic deacetylase. Its substrates include $\alpha$-tubulin, peroxidases, and certain DNA repair proteins $(7,8)$. Recently, HDAC6 was shown to act as a specific deacetylase and E3 ligase to regulate the autophagy-mediated clearance of aggregated proteins and defective mitochondria (9-11). HDAC6 was also suggested to be a tumor suppressor in hepatocellular carcinoma cells (12). HDAC10, another class IIb HDAC, has also been

Authorship note: H. Qian, Y. Chen, and Z. Nian contributed equally to this work. Conflict of interest: The authors have declared that no conflict of interest exists. Submitted: December 9, 2015; Accepted: January 19, 2017.

Reference information: J Clin Invest. 2017;127(4):1353-1369.

https://doi.org/10.1172/JCl85963. shown to regulate autophagy in neuroblastoma cells (13). P300/ CBP-associated factor (PCAF), a HAT, has an important role in DNA damage and certain metabolic pathways (14). Despite our growing understanding of the scope of protein acetylation and its biological functions, the precise mechanism by which regulatory signals or nutrients control protein acetylation and its downstream metabolic consequences remains elusive. High extracellular glucose levels regulate the acetylation and activity of many metabolic enzymes in the glycolysis pathway (15-18). The role of fatty acids (FAs), a major nutritional component, in regulating acetylation and its downstream metabolic response remains unknown.

Obesity is characterized by excessive triacylglycerol (TAG) storage in adipose tissue and is a major risk factor for the development of other metabolic disorders, including diabetes, fatty liver disease, and cardiovascular disease $(19,20)$. The main cellular basis of obesity is the increased number of adipocytes (hyperplasia) and the increased size of adipocytes (hypertrophy). Adipocyte hypertrophy in adults was shown to be the dominant mechanism of adult fat mass expansion $(21,22)$. Adipocyte hypertrophy mainly results from the uncontrolled growth of lipid droplets (LDs) in the presence of excessive FAs (21). Local synthesis of TAG by LD- 
A

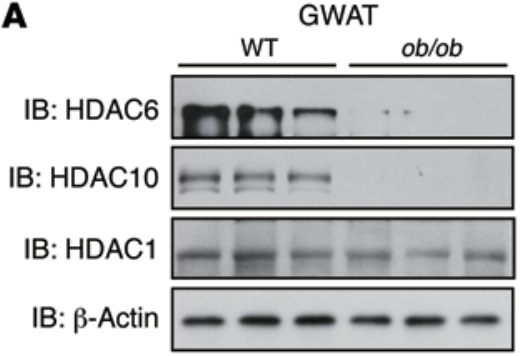

C

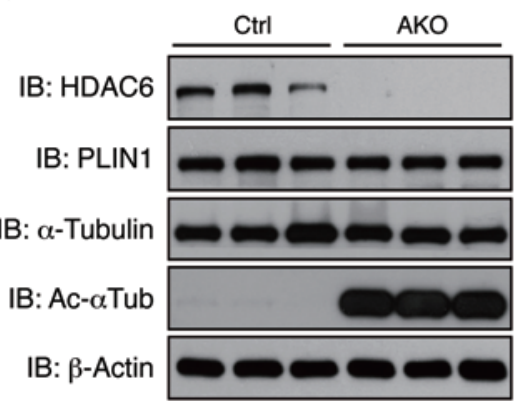

B

Rhesus monkey visceral fat

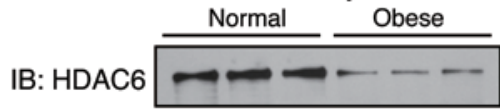

IB: HDAC10

IB: HDAC1

IB: $\beta$-Actin $-\ldots-\ldots$
Human intra-abdominal fat

IB: HDAC6 Normal Obese

IB: HDAC10

IB: HDAC 1

IB: $\beta$-Actin
D

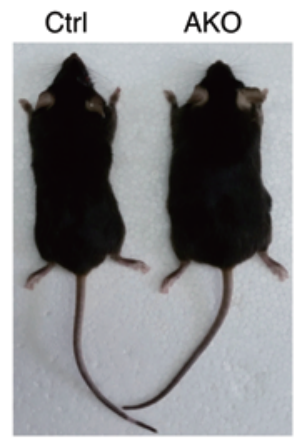

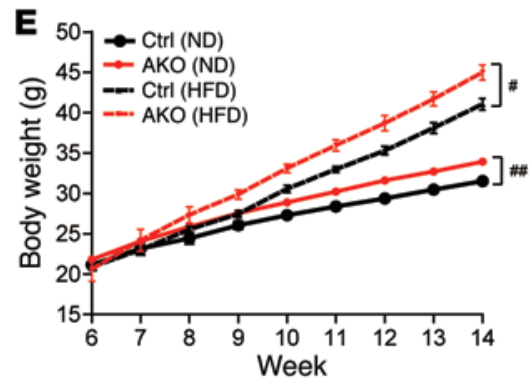

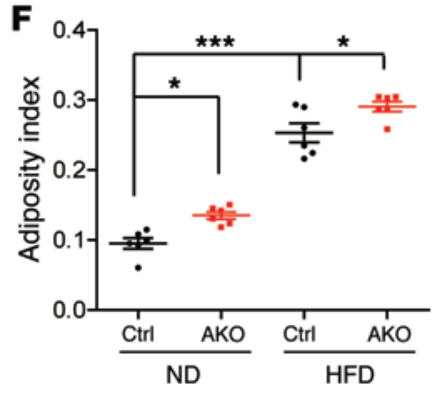

G

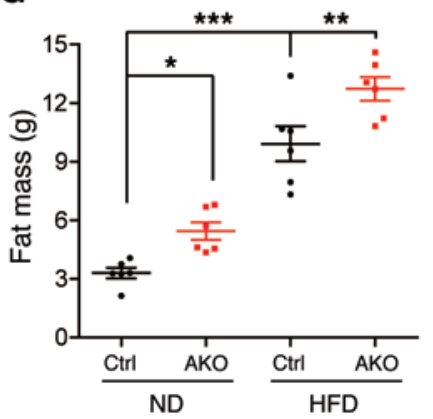

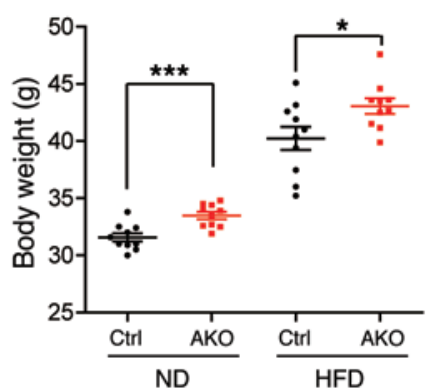

IB: HDAC6

IB: UCP1

IB: $\alpha$-Tubulin

IB: Ac- $\alpha$ Tub

IB: $\beta$-Actin

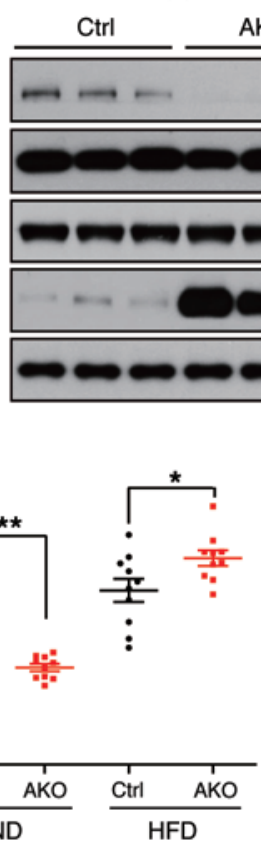

AKO

(and
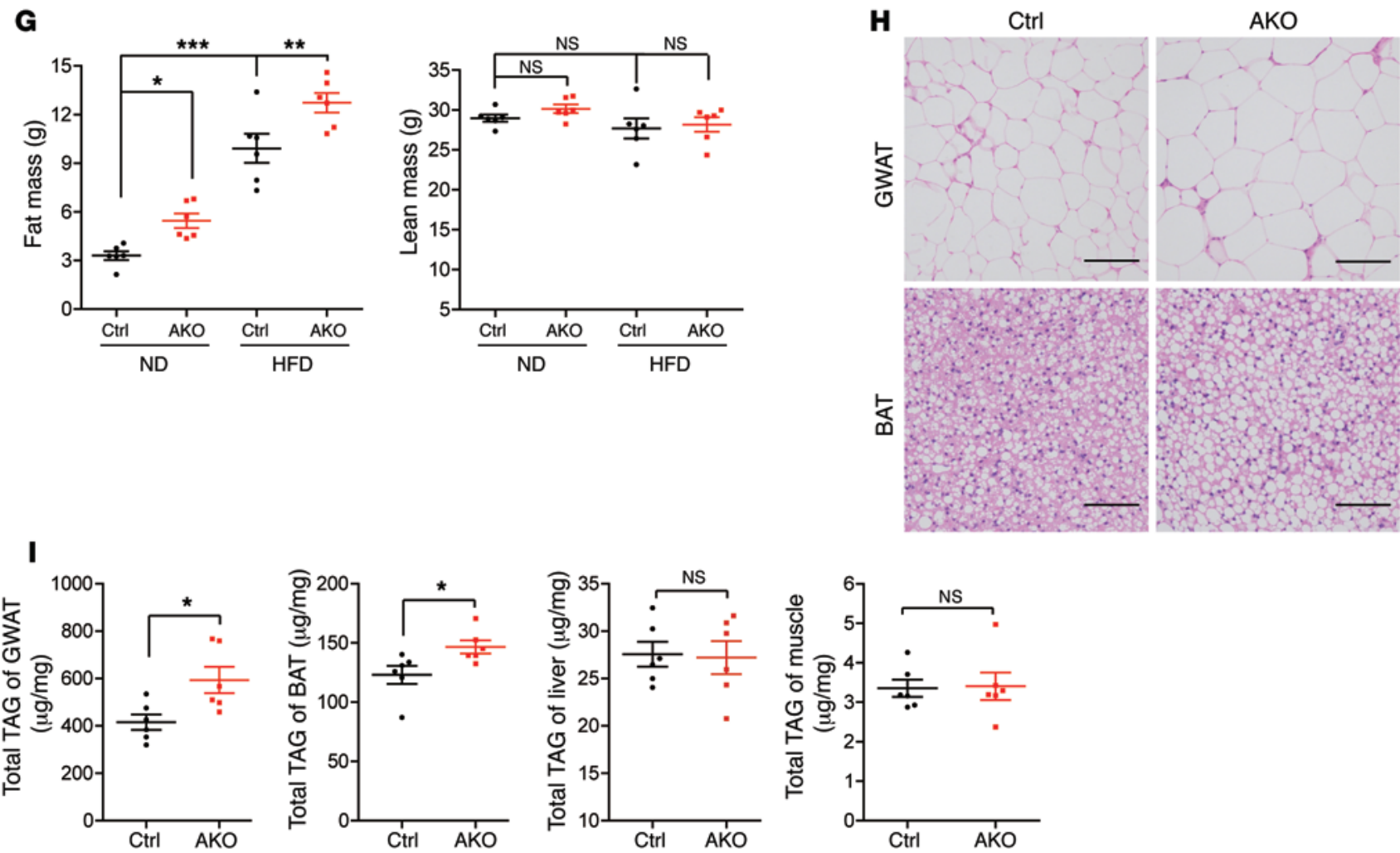
Figure 1. HDAC6 is a negative regulator of lipid storage. (A) Levels of HDAC6 and HDAC10 protein were decreased in the CWAT of leptindeficient (ob/ob) mice ( $n=3$ mice per group). (B) Reduced expression of HDAC6 and HDAC10 in the visceral fat of obese rhesus monkeys and the intra-abdominal fat of humans ( $n=3$ samples per group). (C) Levels of HDAC6 protein in the GWAT and the BAT of control (Ctrl) and Hdac6 AKO mice ( $n=3$ mice per group). Ac- $\alpha$ Tub, acetlyated $\alpha$-tubulin. (D) Representative photograph of 4-month-old control and Hdac6 AKO mice. (E) Growth curve ( $n=6$ mice per group) reflected by body weight analysis ( $n=10$ mice per group) for control and the Hdac6 AKO mice fed ND or HFD. Two-way repeated-measures ANOVA was used to evaluate the data (left panel); ${ }^{\#} P<0.05, \# \#<0.01$. For the for right panel, 2 -tailed Student $t$ test was used; ${ }^{*} P<0.05,{ }^{*} P<0.01,{ }^{* *} P<0.001$. (F) Adiposity index for control and Hdac6 AKO mice ( $n=6$ mice per group). (G) Fat mass and lean mass by MRI scanning of control and Hdac6 AKO mice ( $n=6$ mice per group). (H) Morphology of the GWAT and BAT of control and Hdac6 AKO mice $(n=3$ mice per group). Scale bars: $50 \mu \mathrm{m}$. (I) Total TAG levels of GWAT, BAT, liver, and muscle of control and Hdac6 AKO mice ( $n=6$ mice per group). Data represent the mean $\pm \mathrm{SEM}$; ${ }^{*} P<0.05,{ }^{* *} P<0.01$, ${ }^{* *} P<0.001$, by 2 -tailed Student's $t$ test. One-way ANOVA with Dunnett's correction was used for multiple comparisons ( $\mathbf{F}$ and $\mathbf{G}$ ).

associated enzymes (23-25) and CIDEC/FSP27-mediated LD fusion and growth (26-29) have been shown to be the two crucial mechanisms that control adipocyte hypertrophy. Animals deficient in Cidec display markedly reduced lipid storage in their adipose tissue $(30,31)$ and develop a lipodystrophic phenotype $(32,33)$.

Here, we observed that levels of HDAC6 and HDAC10 were drastically reduced in the adipose tissue of obese mice, monkeys, and human patients. Mice with an adipose-specific depletion of Hdac6 displayed increased fat storage and were insulin resistant. Interestingly, we identified CIDEC as a specific downstream target of HDAC6. Acetylation of CIDEC at K56 by PCAF was abolished by HDAC6, resulting in its decreased stability and activity in promoting LD fusion and growth. More importantly, we identified FAs as the specific stimuli that induced CIDEC acetylation by disrupting the interaction between CIDEC and HDAC 6 on the ER. Our observations revealed a regulatory pathway involved in the coordination of nutrient availability, protein acetylation, and cellular lipid metabolic responses.

\section{Results}

HDAC6 is a negative regulator of obesity development. We systematically measured the expression levels of HDAC1-HDAC10 in the gonadal white adipose tissue (GWAT) of WT and leptin-deficient $(o b / o b)$ mice to assess whether the HDAC family of proteins has a functional role in the development of obesity. Levels of class IIb HDAC proteins (HDAC6 and HDAC10) (Figure 1A and Supplemental Figure 1A; supplemental material available online with this article; https://doi.org/10.1172/JCI85963DS1) were markedly reduced in the GWAT of obese mice, whereas levels of other HDACs in the adipose tissue were similar in WT and obese mice (Figure 1A and Supplemental Figure 1B). Levels of Hdac6 and Hdac10 mRNAs were also reduced, although not as dramatically as protein levels (Supplemental Figure 1A). Markedly lower levels of HDAC6 and HDAC10 were also observed in the GWAT of mice fed a high-fat diet (HFD) (Supplemental Figure 1C). Importantly, HDAC6/10 levels were also substantially decreased in the GWAT of obese rhesus monkeys (Figure 1B, left) and in the intra-abdom- inal GWAT of obese patients who had undergone gastric bypass surgery (Figure 1B, right; Tables 1 and 2; and Supplemental Figure $1, \mathrm{D}$ and $\mathrm{E})$. These data indicate that class IIb HDACs negatively correlated with excess energy-induced lipid storage and the development of obesity.

We generated mice with a conditional Hdac6 allele (Hdac $\left.6^{\text {f/ft}}\right)$ and crossed them with adiponectin-Cre mice to generate an adipocyte-specific knockout of Hdac6 (i.e., Hdac6 AKO) to confirm that HDAC6 indeed has a negative role in the development of obesity. An analysis of HDAC6 protein levels indicated that the deletion was specific to white adipose tissue (GWAT) and brown adipose tissue (BAT) (Figure 1C); it was not observed in the liver (Supplemental Figure 1F). Levels of acetylated $\alpha$-tubulin, a well-characterized downstream target of HDAC6, were markedly increased in the BAT and GWAT of Hdac6 AKO mice (Figure 1C), further confirming the depletion of Hdac6. Hdac6 AKO animals appeared to be obese upon gross morphological examination (Figure 1D). Body weights of Hdac6 AKO mice were increased compared with those of control mice under both normal diet (ND) and HFD conditions (Figure 1E). Weights of the fat pads from several anatomical locations (Tables 3 and 4) and adiposity indexes (Figure 1F) were higher in Hdac6 AKO mice. In addition, total fat mass measured by MRI was increased in animals with adipocytespecific knockout of $H d a c 6$, although total lean mass was similar in control and AKO mice under both ND and HFD conditions (Figure 1G). LD size and total amount of TAG in the GWAT and BAT were both increased in the Hdac6 AKO mice (Figure 1H), whereas total amounts of TAG in the liver and muscle were similar in control and Hdac6 AKO mice (Figure 1I and Supplemental Figure 1G). These data indicate that $H d a c 6$ deficiency in adipose tissue results in the accumulation of more lipids and the development of obesity.

We performed metabolic cage experiments using 8 mice from each control and Hdac6 AKO group to further evaluate the physiological role of HDAC6 in controlling lipid storage. We observed significantly reduced oxygen consumption in Hdac6 AKO mice using a direct comparison method (Figure 2A), a ratio-based method (Supplemental Figure 2A), and analysis of covariance (ANCOVA) (Supplemental Figure 2B). Respiration exchange rate (Figure 2B) and energy expenditure (Figure 2C) were also lower in Hdac6 AKO mice. No differences in food intake (Supplemental Figure 2C) and physical activity were observed between control and AKO mice (Supplemental Figure 2D). Consistent with the reduced oxygen consumption rate, we observed lower levels of several mitochondrial proteins (CPT1, CPT2, COX4, and cytochrome $c$ ) in the GWAT of Hdac6 AKO mice (Figure 2D and Supplemental Figure 2E). Levels of COX4 and cytochrome $c$ in the BAT of AKO mice were also reduced (Figure 2E). Next, we observed similar levels of BAT mitochondrial DNA in control and Hdac6 AKO mice, indicating that their BAT contained similar numbers of mitochondria (Supplemental Figure 2F). Furthermore, we directly evaluated the function of mitochondria in the BAT of control and Hdac6 AKO mice by isolating their mitochondria and measuring the oxygen consumption rate by use of a XFe96 Seahorse analyzer (Seahorse Bioscience). We observed an approximately $20 \%$ reduction in basal respiration, ADP-stimulated respiration, and maximal respiration after the addition of the uncoupler FCCP in the BAT mitochondria of Hdac6-deficient compared with control mice (Figure 2F). The 
Table 1. Information on normal and obese rhesus monkeys

$\begin{array}{lcccccccccc}\text { ID } & \text { Type } & \text { Sex } & \text { Age } & \text { BW }(\mathbf{k g}) & \text { Glucose }(\mathbf{m m o l} / \mathbf{l}) & \text { Insulin }(\boldsymbol{\mu U} / \mathbf{l}) & \text { TAG (mmol/l) } & \text { CE (mmol/l) } & \text { HDL (mmol/l) } & \text { LDL (mmol/l) } \\ 99013 & \text { Normal } & \text { Male } & 13.8 & 8.14 & 4.68 & 17.90 & 2.66 & 0.47 & 1.26 & 0.99 \\ 920653 & \text { Normal } & \text { Male } & 19.8 & 8.24 & 4.09 & 10.87 & 3.67 & 0.28 & 1.43 \\ 921101 & \text { Normal } & \text { Male } & 18.9 & 12.42 & 4.12 & 14.25 & 2.79 & 0.36 & 1.54 \\ 950805 & \text { Obese } & \text { Male } & 15.7 & 22.30 & 4.04 & 32.95 & 3.29 & 0.48 & 1.48 & 1.20 \\ 950607 & \text { Obese } & \text { Male } & 14.9 & 20.70 & 4.64 & 30.09 & 3.62 & 0.85 & 1.60 \\ 950723 & \text { Obese } & \text { Male } & 15.2 & 20.10 & 4.15 & 38.09 & 2.51 & 0.52 & 1.57 & 0.83\end{array}$

$\mathrm{CE}$, cholesteryl ester.

expression levels of white fat browning genes (Prdm16, Cidea, Ucp1, and Dio2), genes in the TAG synthesis pathway (Acsl3, Agapat4, Gpat4, Lpin1, Dgat1, and Dgat2), and genes in the FA biosynthesis pathways (Fas and Acc1) in the GWAT of control and Hdac6 AKO mice were similar (Supplemental Figure 2, G and H). In addition, we observed a similar rate of FA uptake in control and Hdac6-deficient white adipocytes (Supplemental Figure 2I). However, the rate of hormone-stimulated lipolysis was slightly lower in Hdac6 AKO mice (Figure $2 \mathrm{G}$ ). The reduced mitochondrial activity in the BAT and lower lipolysis activity in the animals with adipose-specific depletion of Hdac6 likely contributed to their reduced whole body metabolic activity and increased adiposity index.

To evaluate the insulin sensitivity of animals with adipose tissue-specific knockout of Hdac6, we first performed glucose (GTT) and insulin (ITT) tolerance tests. Both the GTT and ITT showed that Hdac6 AKO mice had lower insulin sensitivity compared with control mice (Supplemental Figure 2J). We then evaluated the insulin sensitivity of Hdac6 AKO mice using a hyperinsulinemic-euglycemic clamp. Consistent with the results of the GTT and ITT, mice with adipocyte-specific knockout of Hdac6 were insulin resistant compared with control mice. This was manifested by a decreased glucose infusion rate (GIR) (Figure $2 \mathrm{H}$ ), lower glucose disposal rate (GDR) (Figure 2I), and lower insulin-stimulated GDR (IS-GDR) (Figure 2J). These animals did not show a change in the ability of insulin to suppress hepatic glucose production (HGP) (Supplemental Figure 2K) and reduce circulating free FA (FFA) levels (Supplemental Figure 2L). These data indicate that deletion of Hdac6 in adipose tissue leads to systemic and skeletal muscle insulin resistance. We found that serum levels of adiponectin were decreased in animals with adipose-specific knockout of Hdac6 (Figure 2K). Further evaluations of the different forms of adiponectin by native gel analysis indicated that levels of both the high-molecular-weight (HMW) and medium-molecular-weight (MMW) forms of adiponectin were decreased in Hdac6 AKO mice (Supplemental Figure 2M). Expression levels of several inflammatory cytokines, including Tnfa and Il18, were also increased in the GWAT of the Hdac6 AKO mice (Supplemental Figure 2N). The reduced secretion of adiponectin and increased expression of inflammatory cytokines may contribute to insulin resistance in Hdac6 AKO mice. Overall, our data strongly suggest that HDAC6 is an important negative regulator of obesity development.

CIDEC is a downstream target of HDAC6. We isolated the microsomal fraction from the GWAT of control and Hdac6 AKO mice to identify the potential downstream target of HDAC6 that mediates its function in controlling lipid storage (34). Proteins were digested with trypsin, and acetylated peptides were enriched with an antibody against acetylated lysine residues. These peptides were subjected to mass spectrometry (MS) analysis by tandem liquid chromatography-tandem MS (LC-MS/MS). CIDEC was identified as one of the potential downstream targets of HDAC6, as its acetylation was increased in the GWAT of Hdac6 AKO mice (see Supplemental material, Data sheet 1). As CIDEC was shown to have an important role in promoting lipid storage by controlling LD fusion, we further evaluated its acetylation by immunoprecipitating acetylated proteins from the GWAT of control and Hdac6 AKO mice using an antibody against pan-acetylated lysine. The immunoprecipitated products were blotted with antibodies against several LD-associated proteins (ADRP, TIP47, PLIN1, and CIDEC). Indeed, we observed dramatically increased acetylation of CIDEC in Hdac6-deficient adipocytes (Figure 3A). No acetylation of ADRP and TIP47 was observed in the GWAT. PLIN1 was detected in the immunoprecipitated product, but its

\section{Table 2. Information on normal and obese individuals}

\begin{tabular}{|c|c|c|c|c|c|c|c|c|c|}
\hline ID & Type & Sex & Age & BMI & Glucose (mmol/l) & TAG (mmol/l) & CE (mmol/l) & $\mathrm{HDL}$ (mmol/l) & $\mathrm{LDL}$ (mmol/l) \\
\hline 1113 & Normal & Male & 48 & 22.10 & 5.50 & 1.46 & 3.93 & 1.09 & 2.30 \\
\hline 1119 & Normal & Male & 64 & 23.10 & 4.30 & 1.23 & 4.95 & 1.02 & 3.01 \\
\hline 1112 & Normal & Male & 64 & 23.20 & 5.00 & 1.30 & 4.30 & 1.06 & 2.00 \\
\hline DS130 & Obese & Male & 46 & 30.02 & 10.12 & 1.92 & 5.00 & 1.10 & 2.98 \\
\hline DS134 & Obese & Male & 48 & 26.35 & 7.18 & 1.08 & 3.23 & 0.87 & 1.78 \\
\hline DS127 & Obese & Female & 59 & 31.64 & 8.63 & 1.97 & 5.23 & 1.37 & 3.14 \\
\hline
\end{tabular}


Table 3. Adipose tissue weight and blood chemistry of control (Ctrl) and Hdac6 AKO mice under ND conditions

ND

\begin{tabular}{cc}
\multicolumn{2}{c}{ Ctrl } \\
$n$ & Mean \pm SEM \\
\hline 8 & $1.40 \pm 0.08$ \\
8 & $1.12 \pm 0.07$ \\
8 & $0.24 \pm 0.03$ \\
8 & $0.28 \pm 0.03$ \\
\hline 8 & $1.31 \pm 0.06$ \\
8 & $0.11 \pm 0.01$ \\
6 & $9.06 \pm 0.21$ \\
6 & $1.55 \pm 0.12$ \\
11 & $0.64 \pm 0.04$ \\
11 & $0.53 \pm 0.04$
\end{tabular}

8

\begin{tabular}{ccc}
\multicolumn{2}{c}{ AKO } & $P$ value \\
$n$ & Mean \pm SEM & \\
8 & $1.83 \pm 0.12$ & $0.003466^{A}$ \\
8 & $1.65 \pm 0.06$ & $0.002558^{A}$ \\
8 & $0.42 \pm 0.04$ & $0.005141^{A}$ \\
8 & $0.37 \pm 0.03$ & $0.034891^{1}$ \\
8 & $1.4 \pm 0.06$ & 0.363280 \\
8 & $0.13 \pm 0.01$ & 0.161311 \\
6 & $9.15 \pm 0.33$ & 0.828478 \\
6 & $1.26 \pm 0.08$ & 0.121785 \\
9 & $0.71 \pm 0.03$ & 0.190201 \\
11 & $0.65 \pm 0.05$ & 0.080436
\end{tabular}

NEFA, non-esterified fatty acids. Data represent mean $\pm \mathrm{SEM} .{ }^{\mathrm{A} P}<0.01,{ }^{\mathrm{B}} P<0.05$, by 2 -tailed Student's $t$ test.

levels were similar in control and Hdac6-deficient GWAT. Levels of acetylated $\alpha$-tubulin, a well-characterized downstream target of HDAC6 used as a positive control, were substantially increased (Figure 3A). Therefore, CIDEC appears to be a downstream target of HDAC6. We ectopically expressed CIDEC in 293T cells to further confirm that CIDEC is a downstream target of HIDAC6 and showed that levels of acetylated CIDEC were increased in cells treated with trichostatin A (TSA), an inhibitor of HDACs, and in cells where Hdac6 was depleted using siRNA (Figure 3B). These data suggest that CIDEC is a specific downstream target of HDAC6 in adipocytes.

PCAF acetylates CIDEC at K56. We coexpressed Flag-tagged CIDEC with different acetyltransferases (i.e., P300, CBP, PCAF, GCN5, and TIP60) in 293T cells to identify the acetyltransferase responsible for CIDEC acetylation. Levels of acetylated CIDEC were specifically increased when it was coexpressed with PCAF, but not when it was coexpressed with other acetyltransferases (Figure 3C). Similar to a previous observation, levels of P53 acetylation were increased when it was coexpressed with all the tested acetyltransferases (Figure 3C). The enzymatically inactive form of PCAF that harbored mutations at its active site (Phe568, Thr569, and Glu570) was not able to acetylate CIDEC (Supplemental Figure 3A). Consistent with it being a downstream target of HDAC6, PCAF-induced CIDEC acetylation was abrogated in the presence of HDAC6 (Figure 3D). This inhibitory effect of HDAC6 on PCAF-induced CIDEC acetylation was reversed in the presence of tubacin, an HDAC6-selective inhibitor, but not in the presence of $\mathrm{NaB}$, a pan-deacetylase inhibitor, further confirming the role of HDAC6 as the deacetylase of CIDEC (Supplemental Figure 3B).

We isolated recombinant MBP-tagged CIDEC and incubated it with recombinant GST-tagged PCAF that contained the catalytic domain (aa 352-832) in vitro to identify the specific lysine residues in CIDEC that could be acetylated by PCAF. CIDEC acetylation was observed in the presence of PCAF (Figure $3 \mathrm{E})$. When the in vitro acetylated CIDEC was subjected to mass spectrometric analysis, several lysine residues on CIDEC were identified as potential acetylation sites (Supplemental Figure 3C). We then generated serial mutations of these lysine residues and coexpressed them with PCAF. Mutations at two lysine residues (i.e., K34R and K56R) completely abolished CIDEC acetylation (Supplemental Figure 3, D-F). A comparison of the amino acid sequences revealed that K56 in CIDEC was conserved across many species (Figure 3F). A single mutation at K34 (i.e., K34R) slightly decreased CIDEC acetylation levels, whereas a mutation at K56 (i.e., K56R) significantly reduced its acetylation (Figure 3F and Supplemental Figure 3, G and H). Furthermore, we used quantitative MS analysis (35) to measure the relative level of acetylated K56 in CIDEC in the GWAT of control and Hdac6 AKO mice. The ratio of the K56-acetylated peptide to the total amount of corresponding peptide (acetylated plus unacetylated peptide) was approximately $0.05 \%$ in control GWAT. In Hdac6-deficient adipose tissue, the ratio of K56 acetylation increased nearly 3-fold, to $0.19 \%$ (Figure $3 \mathrm{G}$ ). We then generated antibodies against the acetylated form of CIDEC (i.e., AcK56) and observed that this antibody was able to specifically recognize acetylated CIDEC in 3T3-L1 adipocytes. Using this antibody, we observed increased CIDEC acetylation at K56 in Hdac6-knockdown cells (Supplemental Figure 3I). Mutations in K56 (i.e., K56Q, acetylation mimic; K56R, acetylation defective) markedly reduced levels of CIDEC acetylation (Figure $3 \mathrm{H}$ ). Furthermore, levels of CIDEC protein and its acetylated form were significantly increased in the GWAT and BAT of Hdac6 AKO mice (Figure 3I). Overall, these data indicate that PCAF acetylates CIDEC at $\mathrm{K} 56$ and HDAC6 promotes CIDEC deacetylation.
Table 4. Adipose tissue weight and blood chemistry of control (Ctrl) and Hdac6 AKO mice under HFD conditions

\begin{tabular}{lccccc} 
HFD & \multicolumn{2}{c}{ Ctrl } & \multicolumn{2}{c}{ AKO } & $P$ value \\
& $\boldsymbol{n}$ & Mean \pm SEM & $\boldsymbol{n}$ & Mean \pm SEM & \\
Subcutaneous fat (g) & 6 & $1.95 \pm 0.16$ & 6 & $2.44 \pm 0.19$ & $0.018930^{A}$ \\
Conadal fat (g) & 6 & $1.51 \pm 0.15$ & 6 & $1.95 \pm 0.14$ & $0.035202^{A}$ \\
Mesenteric fat (g) & 6 & $0.48 \pm 0.07$ & 6 & $0.74 \pm 0.07$ & $0.035904^{A}$ \\
Retroperitoneal fat (g) & 6 & $0.56 \pm 0.04$ & 6 & $0.82 \pm 0.09$ & $0.043120^{A}$ \\
Liver (g) & 6 & $1.61 \pm 0.12$ & 6 & $1.66 \pm 0.11$ & 0.778081 \\
BAT (g) & 6 & $0.10 \pm 0.01$ & 6 & $0.12 \pm 0.01$ & 0.337525 \\
Clucose (mmol/l) & 6 & $10.11 \pm 0.23$ & 6 & $10.34 \pm 0.30$ & 0.753491 \\
Insulin (ng/ml) & 6 & $2.13 \pm 0.19$ & 6 & $2.29 \pm 0.24$ & 0.536759 \\
Serum NEFA (mEq/l) & 6 & $0.61 \pm 0.05$ & 6 & $0.64 \pm 0.03$ & 0.688199 \\
Serum TAG (mg/ml) & 6 & $0.77 \pm 0.08$ & 6 & $0.93 \pm 0.11$ & 0.370233
\end{tabular}

Data represent mean \pm SEM. ${ }^{A} P<0.05$, by 2 -tailed Student's $t$ test. 


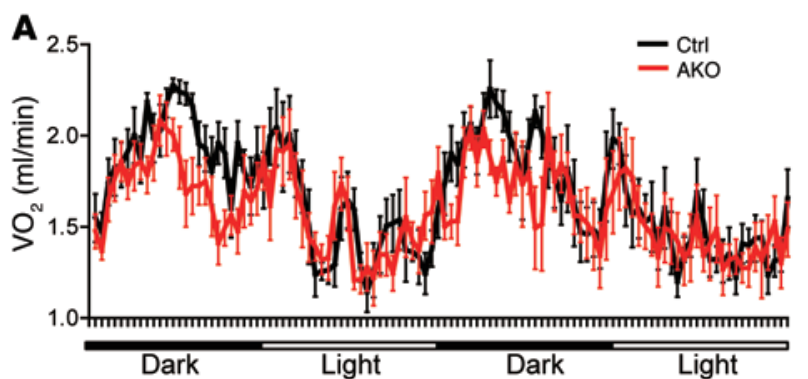

B
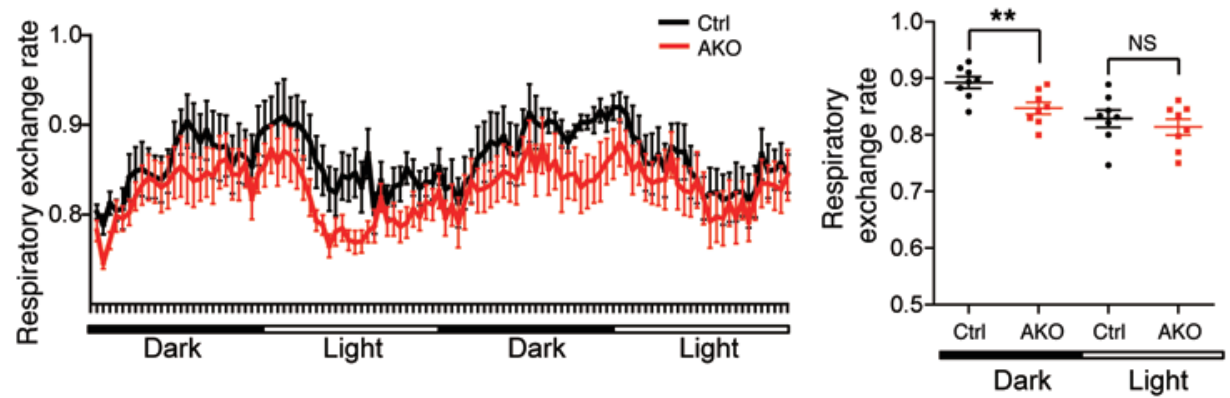

C

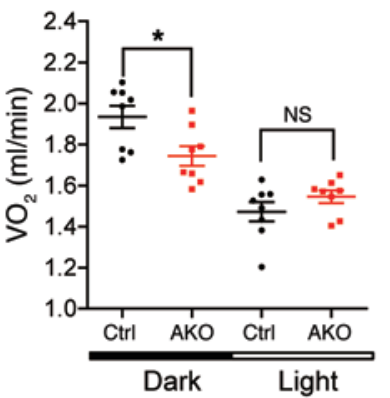

D

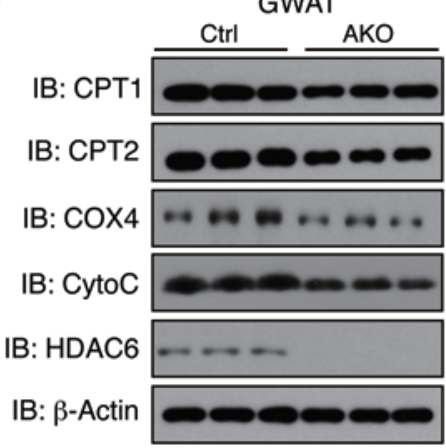

E

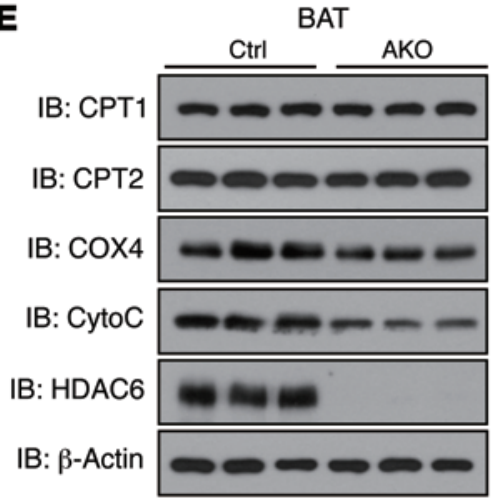

$\mathbf{F}$

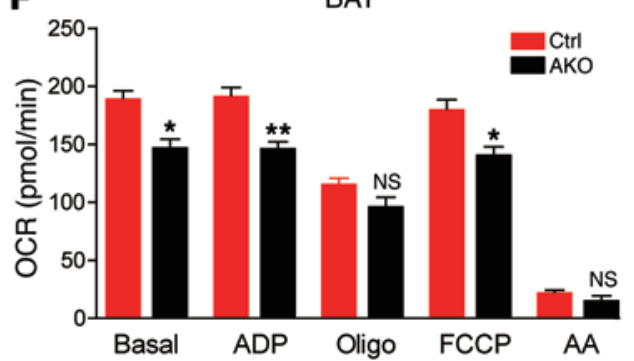

G

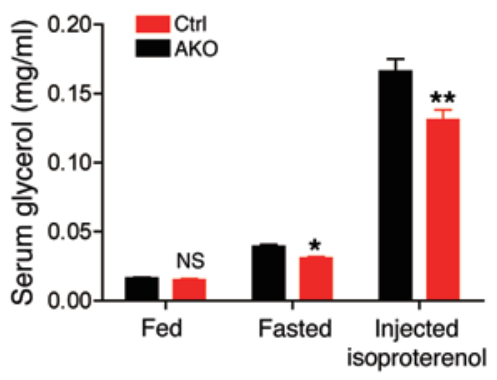

H

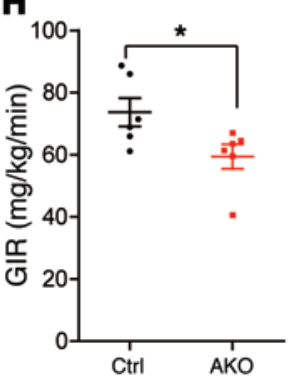

I

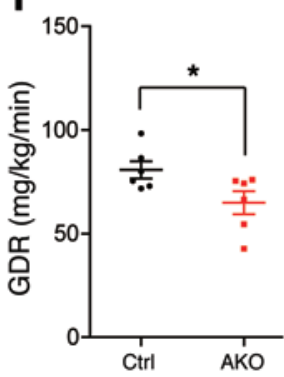

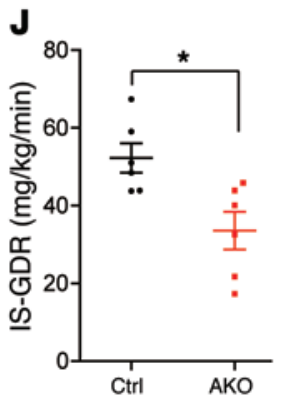

$\mathbf{K}$

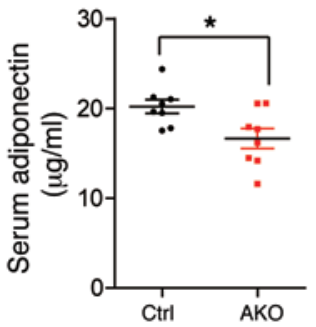

Figure 2. Animals with adipose tissue-specific knockout of Hdac6 have decreased metabolic activity and lower insulin sensitivity. (A) Oxygen consumption ( $\mathrm{VO}_{2}$ ) of control and Hdac6 AKO mice monitored for 48 hours ( $n=8$ mice per group). (B) Respiratory exchange rate analysis according to dark and light phases. From 8pm to 8am (next day) refers to dark phases. From 8am to 8pm refers to light phases. ( $n=8$ mice per group). (C) Energy expenditure analysis of control and Hdac6 AKO mice monitored for 48 hours ( $n=8$ mice per group). (D) Protein expression of CPT1, CPT2, COX4, and cytochrome $c(C y t o C)$, which are important in mitochondrial respiration in the GWAT of control and Hdac6 AKO mice ( $n=3$ mice per group). (E) Protein expression of CPT1, CPT2, COX4, and cytochrome $c$, which are important in mitochondrial respiration in the BAT of control and Hdac6 AKO mice ( $n=3$ mice per group). (F) Quantitative analysis of oxygen consumption rate (OCR) of mitochondria isolated from the BAT of control and Hdac6 AKO mice using Seahorse equipment ( $n=3$ mice per group). (C) Lipolysis in control and Hdac6 AKO mice under fed, fasted, and isoproterenol-stimulated conditions ( $n=6$ mice per group). (H) Levels of GIR of control and Hdac6 AKO mice ( $n=6$ mice per group). (I) Levels of GDR of control and Hdac6 AKO mice ( $n=6$ mice per group). (J) Rate of insulin-stimulated glucose disposal (IS-GDR) of control and Hdac6 AKO mice ( $n=6$ mice per group). (K) Reduced serum levels of adiponectin in control and Hdac6 AKO mice ( $n=6$ mice per group). Data represent mean \pm SEM. ${ }^{*} P<0.05$, ${ }^{* *} P<0.01$, by 2 -tailed Student's $t$ test. 
Acetylation of CIDEC enhances its stability. As we observed increased levels of the CIDEC protein and its acetylated form in Hdac6-deficient adipocytes, we hypothesized that CIDEC acetylation would increase its stability. Therefore, we knocked down Hdac6 in 3T3-L1 adipocytes and examined CIDEC stability. Although CIDEC levels decreased rapidly in WT 3T3-L1 adipocytes (with a half-life of approximately 45 minutes), in the presence of cycloheximide (CHX), which inhibits protein translation, CIDEC was very stable in Hdac6-depleted adipocytes (Figure $4 \mathrm{~A})$. Indeed, the acetylated form of CIDEC was much more stable (Figure 4B). Coexpression of CIDEC with PCAF significantly increased its stability, whereas expression of HDAC6 abrogated PCAF-induced CIDEC stability (Supplemental Figure 4A). The increased CIDEC stability in the presence of PCAF was likely due to its role in inhibiting CIDEC ubiquitination, as we observed reduced levels of CIDEC ubiquitination when it was coexpressed with PCAF (Supplemental Figure 4B). HDAC6 markedly increased CIDEC ubiquitination (Supplemental Figure 4B). To further confirm that CIDEC acetylation enhances its stability, we conducted 3 sets of experiments. First, we tested the stability of the acetylation-mimicking mutant (K56Q) and acetylation-defective mutant (K56R) of CIDEC by ectopically expressing them in 293T cells. We observed that CIDEC K56Q was much more stable than WT CIDEC protein, whereas K56R was less stable (Supplemental Figure 4C). Next, we knocked down HDAC6 in 293T cells using its specific siRNA and then expressed WT CIDEC and K56R in these cells. K56R was less stable than WT CIDEC protein in control cells treated with scramble siRNA. When HDAC6 was knocked down, the stability of CIDEC increased dramatically, as no obvious protein degradation was observed after 1 hour of $\mathrm{CHX}$ treatment. On the contrary, K56R was much less stable than WT protein (Supplemental Figure 4D). Third, we evaluated CIDEC stability by knocking in WT or CIDEC K56R using an adeno-associated virus (AAV) system in Hdac6-deficient adipocytes. WT CIDEC protein in control adipocytes was rapidly degraded, whereas it was very stable in Hdac6-deficient adipocytes. However, CIDEC K56R was degraded much faster in Hdac6-deficient adipocytes compared with WT CIDEC protein (Figure 4C). These data further confirm that HDAC6-mediated deacetylation of CIDEC significantly reduced its stability. Despite reduced protein stability for CIDEC K56R, its ubiquitination levels were similar to those of WT CIDEC (Supplemental Figure 4E), indicating that K56 is not the ubiquitination site. Consistent with the positive role of CIDEC acetylation in controlling its stability, levels of CIDEC protein and its acetylated form were increased in the GWAT of obese mice, monkeys, and human patients (Figure 4, D-F, and Supplemental Figure 4F).

Acetylation of CIDEC enhances $L D$ fusion activity. We then evaluated the function of CIDEC acetylation in controlling lipid storage in adipocytes by measuring LD size in 3T3-L1 adipocytes that were depleted of either Pcaf or Hdac6. 3T3-L1 adipocytes with Pcaf knockdown displayed smaller LDs and lower cellular TAG levels, whereas Hdac6 depletion resulted in the accumulation of more TAGs and large LDs in 3T3-L1 adipocytes (Figure 5, A and B). Lipid exchange activity, a hallmark of CIDEC-mediated LD fusion, was reduced in Pcaf-knockdown 3T3-L1 adipocytes but increased in Hdac6-knockdown adipocytes (Figure 5C). Coexpression of CIDEC and PCAF in 3T3-L1 preadipocytes also resulted in the accumulation of larger LDs, whereas coexpression of CIDEC and HDAC6 led to the accumulation of smaller LDs (Figure 5D). When expressed in 3T3-L1 preadipocytes, the acetylation-defective mutant of CIDEC (K56R) had significantly lower LD fusion activity (Figure 5, E-G). By contrast, cells expressing an acetylation-mimicking mutant of CIDEC (i.e., K56Q) displayed increased rates of lipid exchange and LD fusion, resulting in the accumulation of larger LDs, primarily due to the increased levels of CIDEC K56Q (Figure 5, E-G). Overall, these data indicate that CIDEC acetylation increases its stability and ability to promote LD fusion and lipid storage in adipocytes.

FAs increase CIDEC stability and acetylation. We examined CIDEC levels in mature 3T3-L1 adipocytes treated with various FAs and glucose to evaluate the role of nutrients in regulating CIDEC expression. CIDEC protein (but not RNA) levels were markedly increased in the presence of palmitic acids (PAs) and oleic acids (OAs), but not glucose (Figure 6A and Supplemental Figure 5A). Time course analysis showed that the stability of CIDEC and levels of acetylated CIDEC in mature adipocytes were markedly increased in the presence of OA (Figure 6B and Supplemental Figure 5B). In addition, when ectopically expressed, the relative stoichiometry of acetylated $\mathrm{K} 56$ in CIDEC is $1.75 \%$. In the presence of $\mathrm{OA}$, the ratio was increased to approximately $2.6 \%$, a more than 1-fold increase in K56 acetylation (Supplemental Figure 5C). Levels of endogenous CIDEC acetylation were also increased in OA-treated 3T3-L1 adipocytes (Figure 6C). OA-induced CIDEC acetylation was abolished by the K56R mutation (Supplemental Figure 5D). When PCAF was knocked down in 293T cells using a specific siRNA, OA-induced CIDEC acetylation was decreased compared with that in control cells that were treated with a scrambled siRNA (Figure 6D). Overexpression of HDAC6 completely abolished OA-induced CIDEC acetylation (Figure 6E). Consistent with their roles in enhancing CIDEC acetylation and stability, both OA and TSA reduced CIDEC ubiquitination (Figure 6F). The dosage analysis indicated that the levels of acetylated CIDEC increased as the amount of OA increased (Supplemental Figure 5E). CIDEA, a protein closely related to CIDEC, also showed increased acetylation and stability in the presence of OA and TSA (Supplemental Figure 5, F and G). Overall, these data suggest that CIDEC acetylation is induced by extracellular FAs, and PCAF and HDAC6 mediate FA-induced CIDEC acetylation.

FA induces a dynamic association among CIDEC, PCAF, and HDAC6. We investigated the potential interaction of CIDEC with PCAF and HDACs in 3T3-L1 adipocytes to delineate the underlying mechanism of FA-induced CIDEC acetylation. When CIDEC was pulled down by its antibody, PCAF, HDAC10, and HDAC6 were detected in the immunoprecipitated products, suggesting that PCAF and HDAC6 can interact with CIDEC in adipocytes (Figure 6G). Next, we assessed whether FAs could affect the interaction between CIDEC and PCAF and CIDEC and HDAC6 in mature 3T3-L1 adipocytes that were cultured under starvation conditions (to reduce extracellular lipid supply) and then with the addition of OA. Under starvation condition, an association between CIDEC and HDAC6 was detected, whereas the association between CIDEC and PCAF was reduced (Figure 6H). In the presence of OA, the amount of HDAC6 that associated with CIDEC gradually decreased, whereas the amount of PCAF that associated 
A

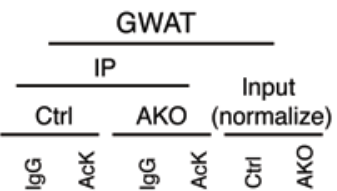

IB: CIDEC

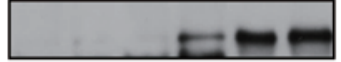

IB: PLIN1

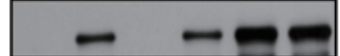

IB: ADRP

IB: TIP47

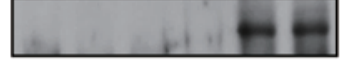

B: $\alpha$-Tubulin

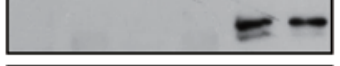

$\lg G$
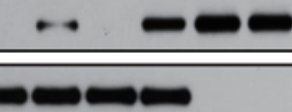

B

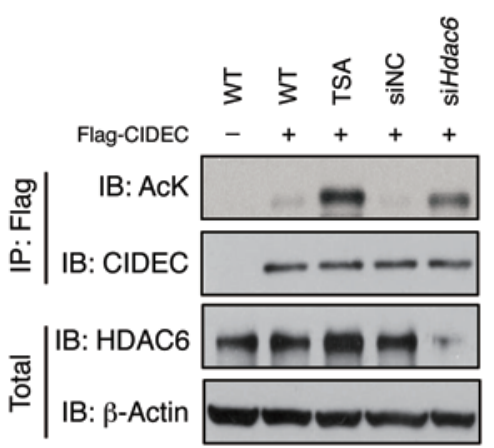

C

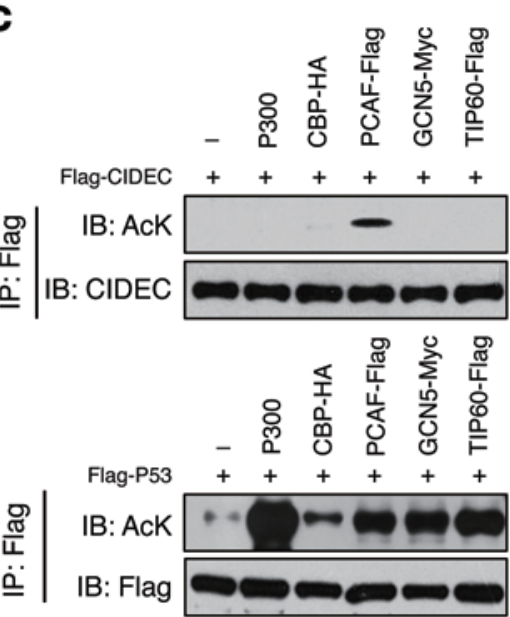

D

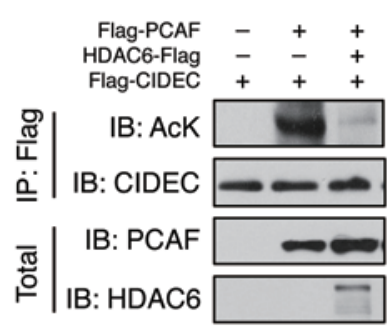

G

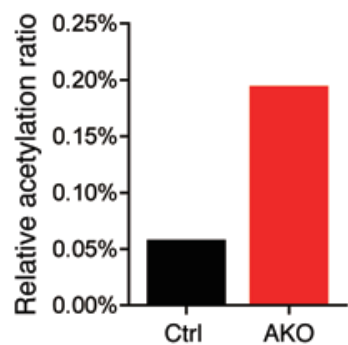

H

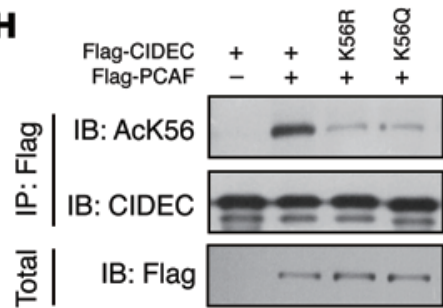

E GST-PCAF (352-832) ++-+
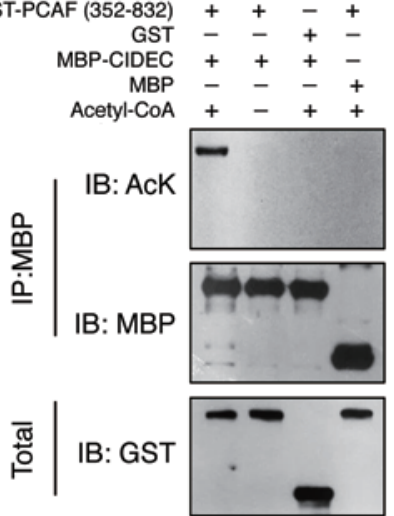

$\mathbf{F}$

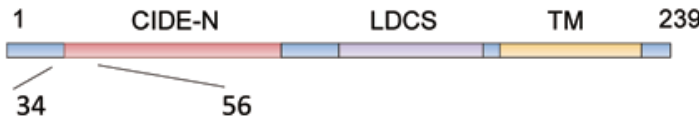

30 -LVSKPSRET-KVRKGIM-59 Mus musculus

30 -LLSEPSPKA-SVRKGIM-59 Homo sapiens

30 -LLSESSQEA-SVRKGIM-59 Mustela putorius furo

26 -TQCLLSRPA-SLRKGIM-55 Xenopus laevis

30 -LLSEPSLEA-SVRKGIM-59 Sus scrofa
I

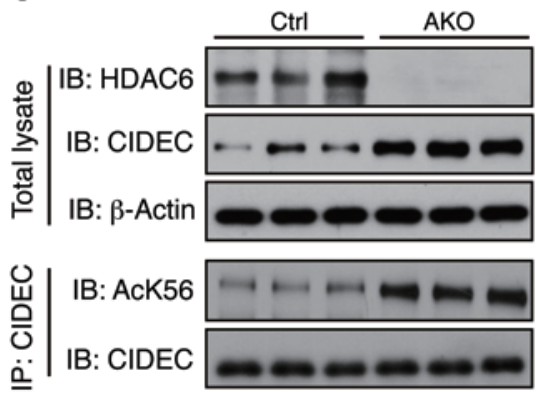

GWAT

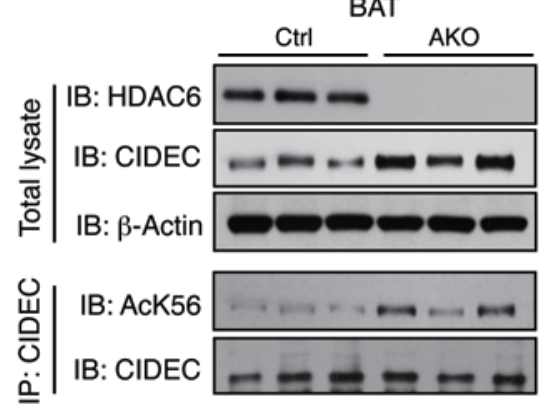

Figure 3. CIDEC is deacetylated by HDAC6 and acetylated by PCAF at K56. (A) Levels of CIDEC acetylation were increased in the GWAT of Hdac6 AKO mice. Levels of $\alpha$-tubulin were used as a positive control. IgC was used as a loading control. To detect CIDEC acetylation in vivo, the GWAT tissue sample was incubated with $A / G$ beads conjugated to antibodies against acetylated lysine, and the immunoprecipitates were blotted with antibodies against CIDEC and other LD-associated proteins (PLIN1, ADRP, TIP47). Data represent results from at least 3 independent experiments. (B) Ectopically expressed CIDEC was acetylated in the presence of TSA or when Hdac6 was knocked down. Data represent results from at least 3 independent experiments. (C) PCAF acetylates CIDEC. Flag-CIDEC was coexpressed in 293T cells with different acetyltransferases. Upper panel shows levels of CIDEC acetylation; lower panel shows levels of P53 acetylation when Flag-CIDEC and Flag-P53 were coexpressed with various acetyltransferases. Data represent results from at least 3 independent experiments. (D) HDAC6 deacetylates CIDEC. Data represent results from at least 3 independent experiments. (E) PCAF acetylates CIDEC in vitro. Bacterially isolated CIDEC-MBP was incubated with GST-PCAF containing the enzymatic domain (active) in vitro. Data represent results from at least 3 independent experiments. (F) Identification of the conserved $\mathrm{K} 56$ residue in difference species. (C) Ratio of acetylated CIDEC at K56 in the adipose tissue of control and Hdac6 AKO mice. (H) Characterization of anti-acetyl-K56 (AcK56) antibody. Data represent results from at least 3 independent experiments. (I) Increased levels of CIDEC protein and its acetylated form in the GWAT and the BAT of Hdac6 AKO mice. The immunoprecipitated CIDEC was normalized ( $n=3$ mice per group). 
A
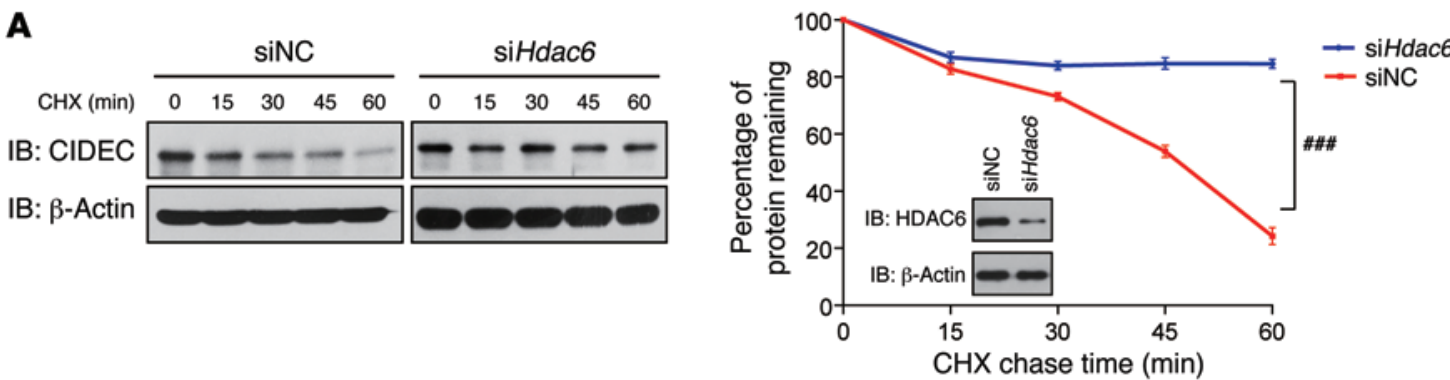

B

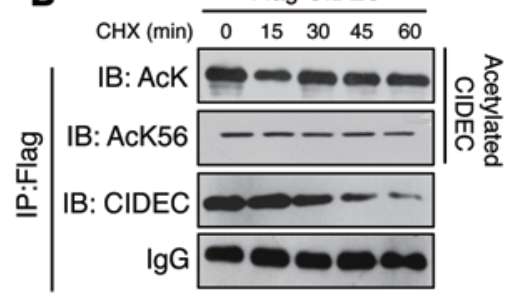

D

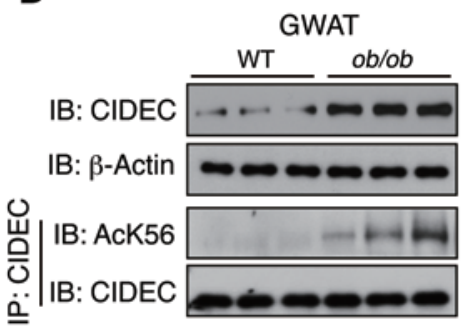

C
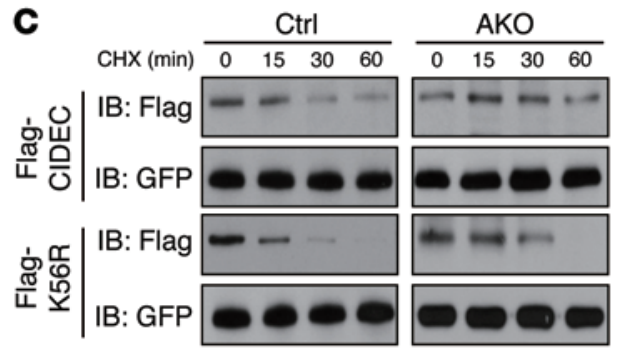

E

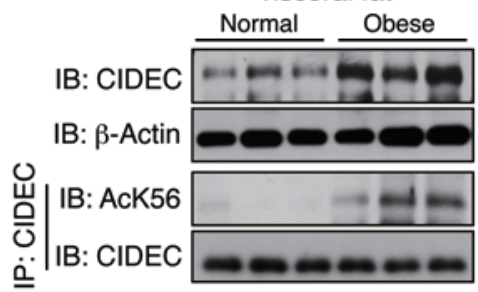

$\mathbf{F}$

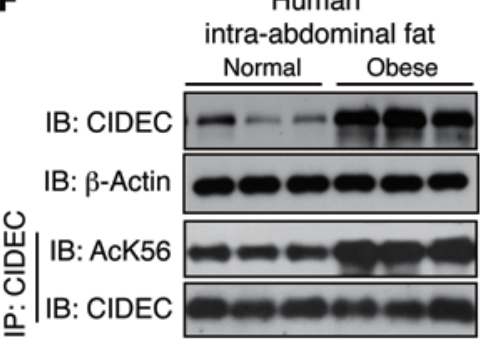

Figure 4. Acetylation of CIDEC enhances its stability. (A) Knockdown Hdac6 increased CIDEC stability in 3T3-L1 adipocytes. Data represent mean \pm SEM . Two-way repeated-measures ANOVA was used to evaluate the data; ${ }^{\# \# ~} P<0.001$. Knockdown efficiency for Hdac6 was determined by Western blot analysis. Data represent results from at least 3 independent experiments. (B) Acetylated CIDEC is more stable. 293T cells were transfected with Flag-tagged CIDEC and treated with CHX at different time points. CIDEC protein was immunoprecipitated using antibodies against FLAC and blotted with antibody against acetylated lysine (AcK56). Data represent results from at least 3 independent experiments. (C) Stability of CIDEC and CIDEC K56R in differentiated stromal vascular fraction (SVF) adipocytes from control and Hdac6 AKO mice. Data represent results obtained from 3 experiments. (D-F) Levels of CIDEC protein and acetylation were both increased in the adipose tissue of mice, monkeys, and humans. The GWAT of WT and leptin-deficient $o b / o b$ mice; visceral fat of normal and obese rhesus monkeys; and intra-abdominal fat of normal and obese human patients were isolated and homogenated. CIDEC protein was immunoprecipitated and normalized using antibody against CIDEC and then blotted with CIDEC or AcK56 antibody ( $n=3$ mice, rhesus monkey, and human samples per group).

with CIDEC increased (Figure 6H). We coexpressed CIDEC and HDAC6 or CIDEC and PCAF in 293T cells grown under FA-free starvation conditions and then with the addition of $\mathrm{OA}$ to further confirm this result. The association between CIDEC and HDAC6 was observed in the absence of OA and gradually decreased when the cells were incubated with increasing amounts of OA (Figure 6I). By contrast, the amount of CIDEC associated with PCAF was markedly increased with increasing concentrations of OA (Figure 6J). An approximately 5-fold-higher affinity between PCAF and CIDEC was observed in the presence of $200 \mu \mathrm{M}$ OA compared with $50 \mu \mathrm{M}$ OA (Figure 6J). Similarly, when the endogenous CIDEC protein was pulled down from cells that were depleted of FAs with an anti-CIDEC antibody, HDAC6 was detected in the immunoprecipitated product (Figure 6K). In the presence of increasing amounts of FAs, the amount of HDAC6 that associated with CIDEC decreased, while the amount of PCAF that associated with CIDEC increased (Figure 6K). Interestingly, the interaction between PCAF and the acetylation-defective mutant K56R was similar to its interaction with the WT protein, whereas the interaction between PCAF and acetylation-mimicking mutant of CIDEC (K56Q) was reduced (Figure 6L). By contrast, the interaction between HDAC6 and the acetylation-defective mutant (K56R) was significantly reduced (Figure 6M). Therefore, FAs enhanced CIDEC acetylation by promoting the dissociation between CIDEC and HDAC6 6 and the association between CIDEC and PCAF.

TAG synthesis is vital for FA-induced CIDEC acetylation. FAs exert their cellular effects in two ways: (a) by binding to FA receptors to control the downstream signaling pathway; and (b) by being transported into cells for esterification to generate TAGs that are incorporated into nascent LDs. We first knocked down several FA receptors, including GPR40, GPR120, and GPR84, in 3T3-L1 adipocytes using their corresponding siRNAs. The depletion of these FA receptors did not affect CIDEC acetylation (data not shown). Then, we tested the effect of FA transport on CIDEC acetylation by knocking down Fabp 4 and $C d 36$, proteins that are responsible for FA transport. When Fabp4 or Cd36 was knocked 

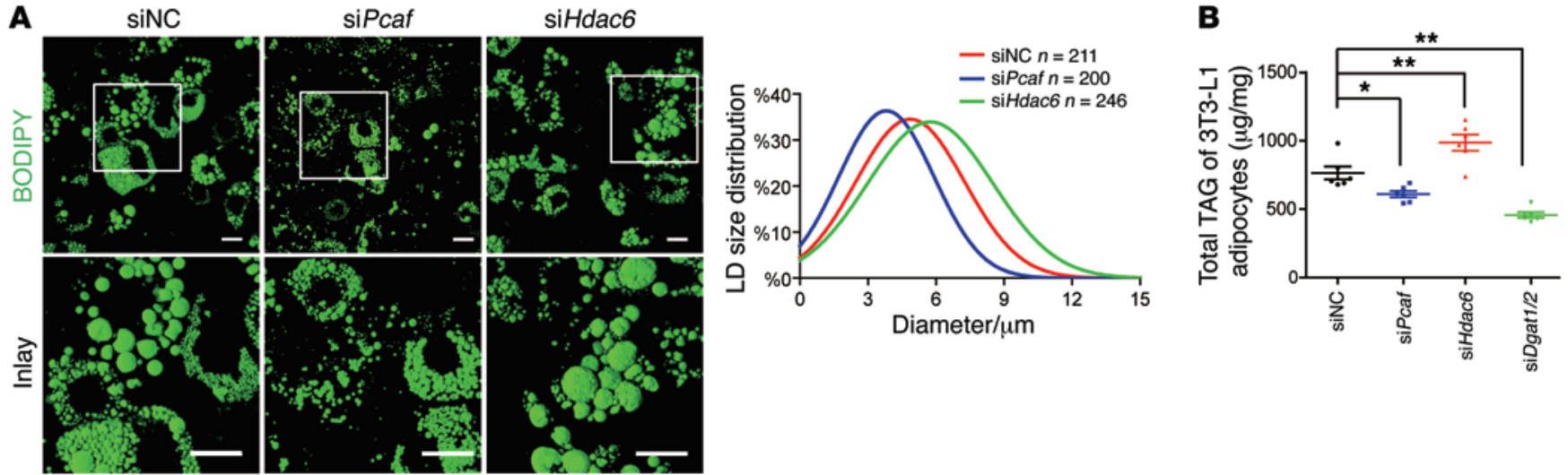

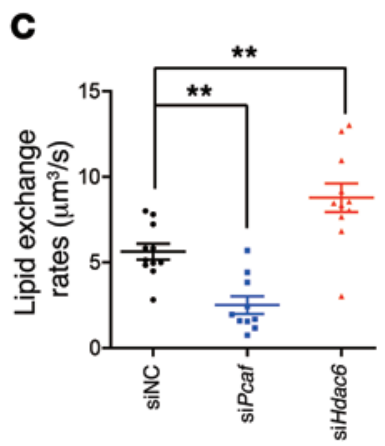

D
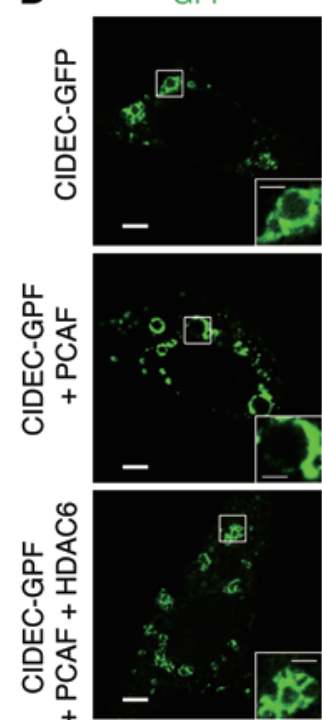

E
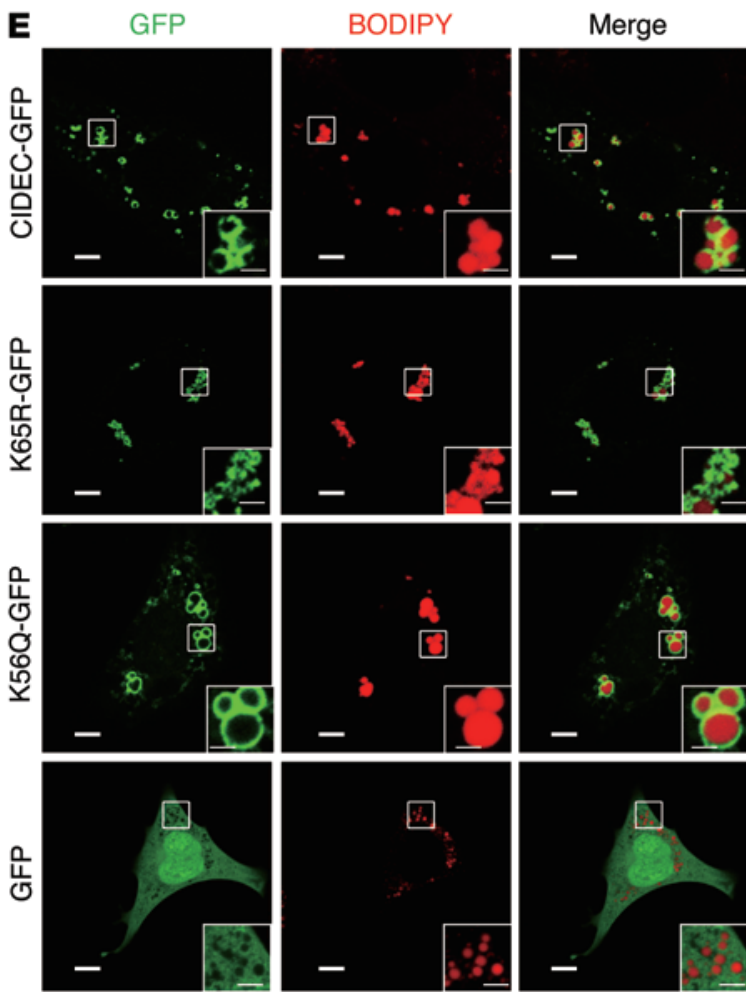
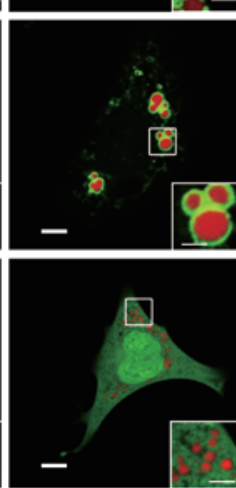

$\mathbf{F}$
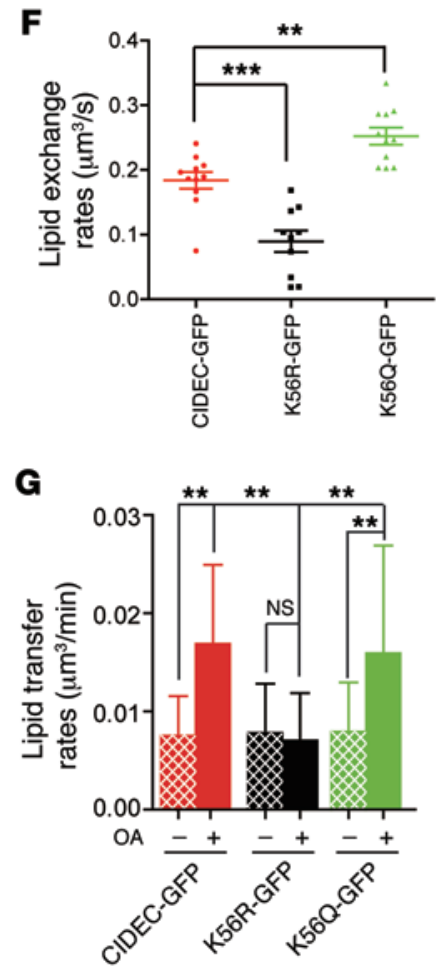

BODIPY
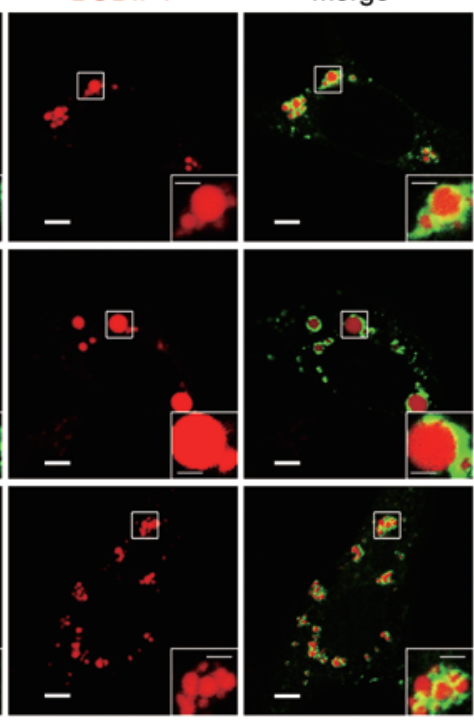

- CIDEC-GFP + PCAF $n=146$ - CIDEC-GFP + HDAC6 $n=165$
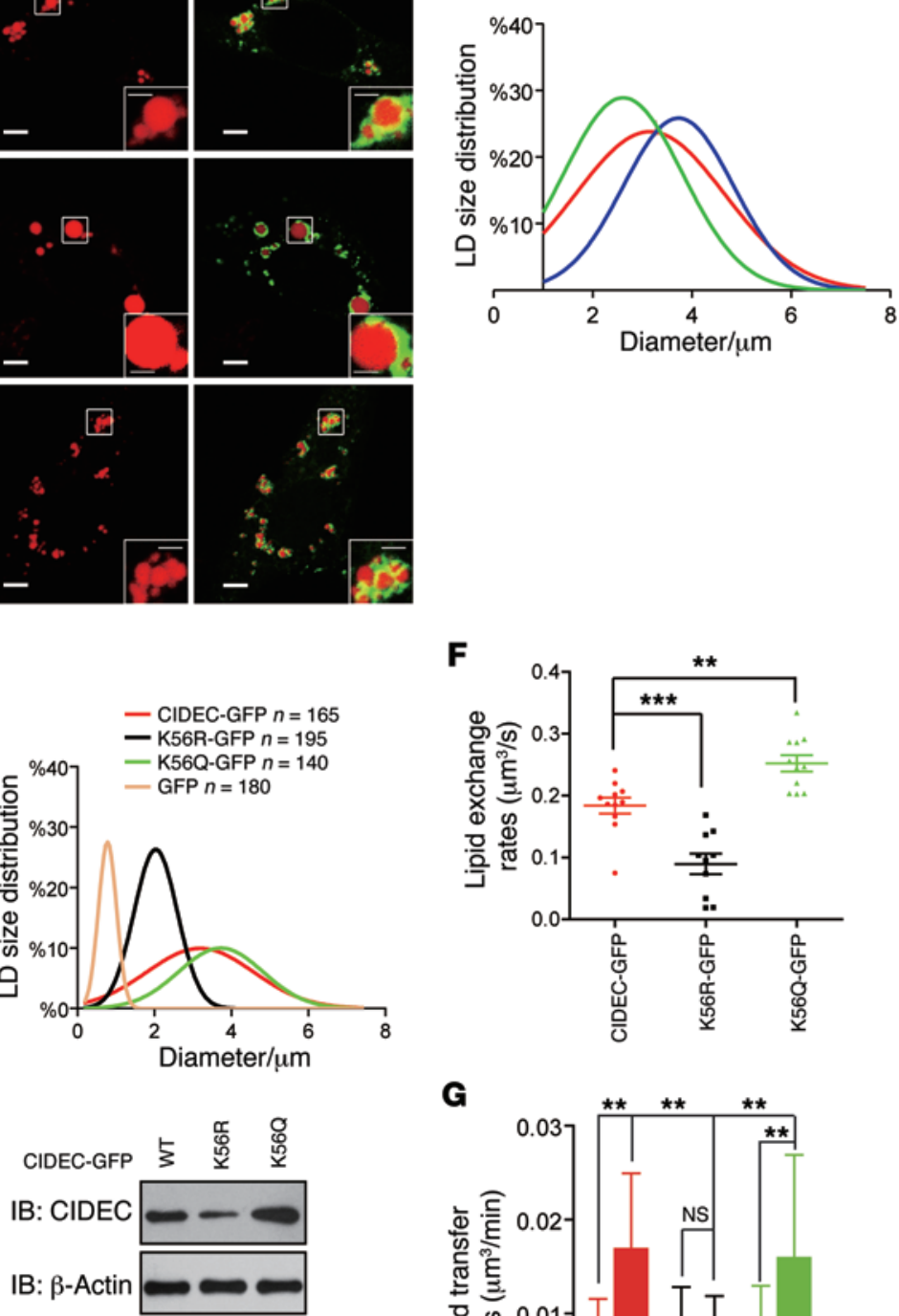
Figure 5. Acetylation of CIDEC enhances LD fusion activity. (A) Knockdown of Pcaf decreased while knockdown of Hdac6 increased LD sizes in 3T3-L1 adipocytes. LDs were labeled with BODIPY 493/503 (green). Scale bars: $20 \mu \mathrm{m}$. The diameter of LDs was obtained from at least 200 cells. (B) Knockdown of Pcaf decreased while knockdown of Hdac6 increased total cellular TAG levels in 3T3-L1 adipocytes ( $n=6$ independent samples per group). (C) Higher lipid exchange activity in Hdac6-knockdown 3T3-L1 adipocytes and lower lipid exchange activity in Pcaf-knockdown adipocytes. Data represent results obtained from at least 10 pairs of LDs per group. (D) Coexpression of CIDEC with PCAF or HDAC6 affects LD sizes. Scale bars: 5 $\mu \mathrm{m}$ and $2 \mu \mathrm{m}$ (insets). Data represent results obtained from at least 140 cells per group. (E) Overexpression of the acetylation-mimicking mutant of CIDEC (K56Q) increases LD sizes in 3T3-L1 preadipocytes. Western blots showing the protein level of CIDEC mutants. Scale bars: $5 \mu \mathrm{m}$ and $2 \mu \mathrm{m}$ (insets). Data represent results obtained from at least 140 cells per group. (F) Overexpression of CIDEC K56Q enhances lipid exchange rate. Data represent results obtained from at least 10 pairs of LDs per group. (G) Overexpression of CIDEC K56Q enhances LD fusion activity. Data represent results obtained from at least 11 pairs of LDs per group. Data represent mean $\pm \mathrm{SEM}$; ${ }^{*}<<0.05,{ }^{* *} P<0.01,{ }^{* *} P<0.001$, by 2 -tailed Student's $t$ test. One-way ANOVA with Dunnett's correction was used for multiple comparisons (B, C, F, and $\mathbf{G}$ ).

down, levels of acetylated CIDEC were markedly reduced in adipocytes in the presence of OA (Figure 7A, Fabp 4 knockdown; and Supplemental Figure 6A: $C d 36$ knockdown, $P<0.01)$. Consistent with this finding, the CIDEC-HDAC6 interaction was increased, whereas the CIDEC-PCAF interaction was decreased (Figure $7 \mathrm{~A})$, suggesting that intracellular FA transport was required for CIDEA acetylation. Next, we checked whether TAG synthesis was required for FA-induced CIDEC acetylation by adding 2-BrO, which blocked the last step of TAG synthesis by inhibiting DGAT activity. Intriguingly, when TAG synthesis was blocked by 2-BrO, the levels of acetylated CIDEC and the association between PCAF and CIDEC were substantially reduced (Supplemental Figure 6B). However, the interaction between CIDEC and HDAC6 was increased (Supplemental Figure 6B). Consistent with this observation, the depletion of Dgat1 and Dgat2 in adipocytes using their corresponding siRNAs also resulted in a decreased association between CIDEC and PCAF but an enhanced association between CIDEC and HDAC6 (Figure 7B). The depletion of several other enzymes (ACSL3, GPAT4, AGPAT4, and lipin 1) in the TAG synthesis pathway also reduced CIDEC acetylation (data not shown). By contrast, coexpression of CIDEC with DAGT1, DAGT2, or AGPAT4 markedly increased CIDEC acetylation (Supplemental Figure 6C). We obtained mice deficient in lipin 1 (an enzyme in the TAG synthesis pathway) and tested the effect of defective TAG synthesis on controlling CIDEC acetylation in a lipodystrophic model to further confirm that TAG synthesis is required for FAinduced CIDEC acetylation. Consistent with a previous observation, lipin 1-deficient animals displayed a typical lipodystrophic phenotype, with very low body fat accumulation (Supplemental Figure 6D). Importantly, we observed lower levels of the CIDEC protein and its acetylated form in the adipose tissue of lipin 1-deficient compared with WT mice. Lipin 1 deficiency did not affect PCAF and HDAC6 protein levels in the GWAT (Figure 7C). Therefore, intracellular FA trafficking and the conversion of FAs to TAG are required for disruption of the interaction between CIDEC and HDAC6 and the accumulation of acetylated CIDEC.
$E R$ is the regulatory site for FA-induced CIDEC acetylation. We biochemically isolated ER-enriched microsomal, LD, and nuclear fractions from 293T cells expressing CIDEC in the presence or absence of OA to determine the site that regulates FA-induced CIDEC acetylation. Endogenous HDAC6 was detected in the cytosolic fraction in the absence of CIDEC. Endogenous PCAF was predominantly detected in the nuclear fraction, with lower amounts in the cytosolic and ER fractions (data not shown). Interestingly, when coexpressed with CIDEC, HDAC6 was detected in the ER fraction in the absence of OA but displayed a reduced ER association in the presence of increasing amounts of OA (Figure 7D and Supplemental Figure 6E), whereas the amount of PCAF that associated with CIDEC was increased in the ER fraction in the presence of OA (Figure 7E).

In mature 3T3-L1 adipocytes, PCAF was detected in the ER fraction, and its association with CIDEC was increased in the presence of OA. HDAC6 was also present in the ER fraction, but it was not readily detectable when the adipocytes were incubated with OA. Levels of the CIDEC protein and its acetylated form were markedly increased in the ER fraction in the presence of OA (Figure 7F). Intriguingly, neither PCAF nor HDAC6 was detected in the LD fractions, despite the presence of higher levels of CIDEC and its acetylated form (Figure 7F). Consistent with this finding, when treated with MG132, increased levels of ubiquitinated CIDEC were detected in the ER fraction, but not the LD fraction (Supplemental Figure 6F), whereas the levels of ubiquitinated CIDEC in the ER were decreased when adipocytes were treated with OA (Supplemental Figure 6G).

We assessed the subcellular localization of CIDEC in the presence or absence of OA by immunofluorescence staining to further confirm the localization of CIDEC, PCAF, and HDAC6. The majority of CIDEC (approximately 80\%) exhibited a diffuse cytosolic pattern in cells grown under starvation conditions (Supplemental Figure $6 \mathrm{H}$ ). In the early course of FA treatment, CIDEC was localized to the ER; then, the association of CIDEC with LD rapidly increased 1 hour after FA treatment (Supplemental Figure 6H). Although PCAF was predominantly localized to the nucleus, PCAF localization to the ER was evident and not dependent on the presence of OA (data not shown) or CIDEC expression (Supplemental Figure 6I). However, HDAC6 alone exhibited a diffuse cytosolic distribution in the absence or presence of FAs (data not shown). When coexpressed with CIDEC, HDAC6 showed a transient but specific colocalization to the ER 30 minutes after FA treatment (Figure 7G). Overall, these data strongly indicate that the ER is the regulatory site of FA-induced CIDEC acetylation.

\section{Discussion}

CIDEC, along with CIDEA and CIDEB, constitute the CIDE family of proteins, which play a vital role in the development of obesity and hepatic steatosis by controlling LD fusion and lipid storage. Here, we revealed that HDAC6 is a major regulatory factor for obesity development. Moreover, the interplay among PCAF, HDAC6, and CIDEC constitutes an evolutionarily conserved regulatory circuit that senses FA levels and controls lipid storage, and its dysregulation leads to the development of obesity and insulin resistance.

First, our analyses indicate that protein levels of class IIb HDACs (HDAC6 and HDAC10) were markedly reduced in the 
A

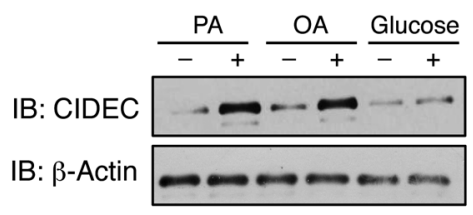

B

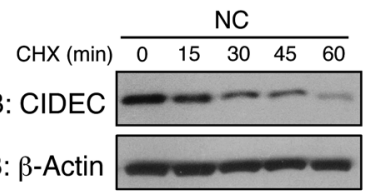

C

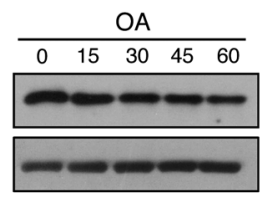

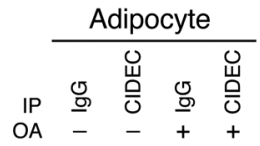

$\underline{\underline{B}}$

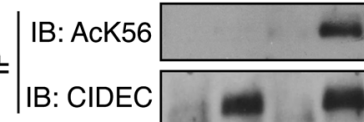

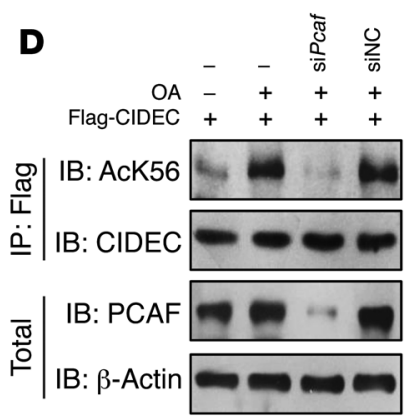

E

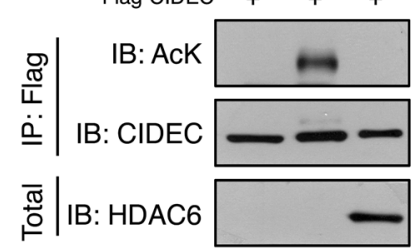

G

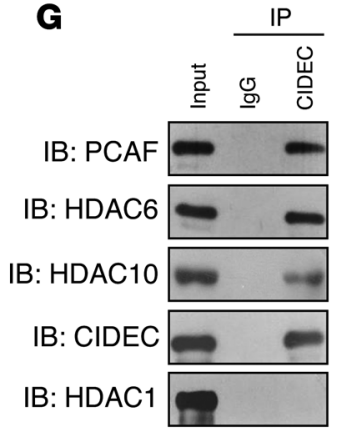

H<smiles>[124IH]</smiles>

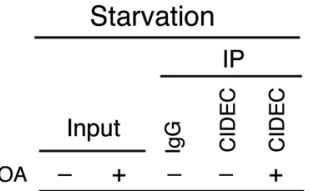

$\mathrm{OA}$

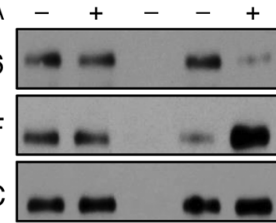

I

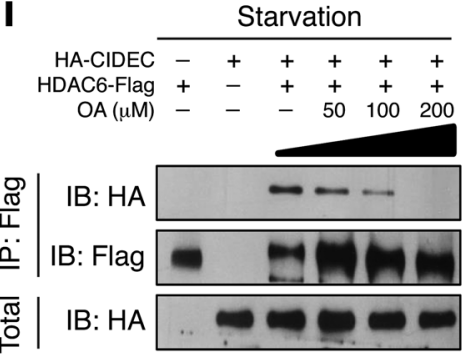

$\mathbf{F}$

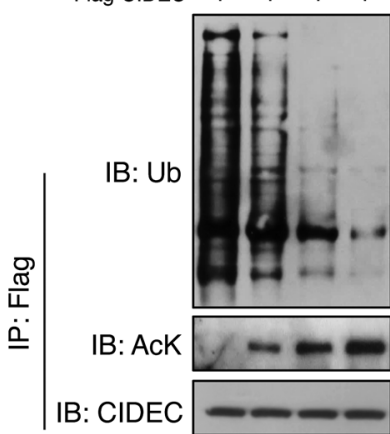

IB: Ub

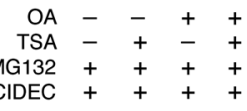

J

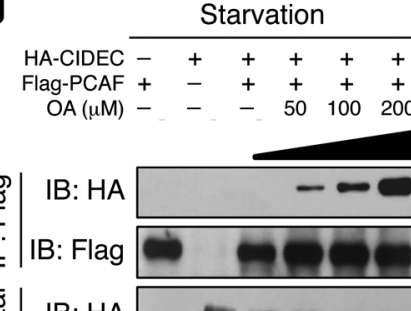

$\mathbf{K}$

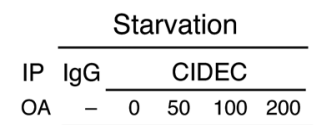

$\stackrel{\bigcup}{\breve{O}}$
$\stackrel{0}{0}$
$\stackrel{0}{=}$

$O A-\square \quad 50 \quad 100 \quad 200$

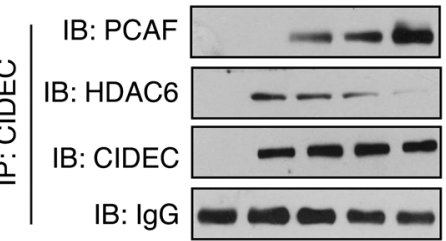

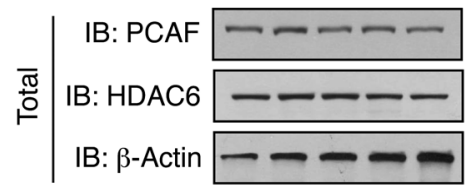

$\mathbf{L}$

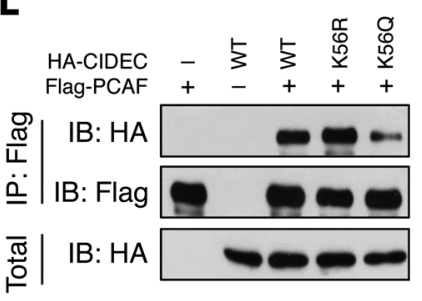

M

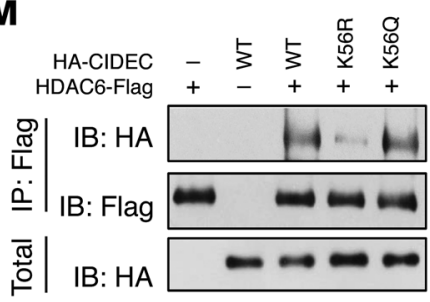

Figure 6. FA induces CIDEC acetylation and dynamic association among CIDEC, PCAF, and HDAC6. (A) FAs increase CIDEC protein levels. Data represent results from at least 3 independent experiments. (B) OA enhances the stability of endogenous CIDEC in 3T3-L1 adipocytes. Data represent results from at least 3 independent experiments. NC, negative control that without OA treatment. (C) OA-induced CIDEC acetylation was at the K56 residue (AcK56) in 3T3-L1 adipocytes. Data represent results from at least 3 independent experiments. (D) Knockdown of Pcaf abolished OA-induced CIDEC acetylation. Data represent results from at least 3 independent experiments. (E) OA-induced CIDEC acetylation was inhibited by the expression of HDAC6. Data represent results from at least 3 independent experiments. (F) Levels of CIDEC ubiquitination (Ub) were decreased in the presence of OA or TSA. Data represent results from at least 3 independent experiments. (G) Interaction among CIDEC, PCAF, HDAC6, and HDAC10 in differentiated 3T3-L1 adipocytes. Data represent results from at least 3 independent experiments. (H) OA inhibited the interaction between CIDEC and HDAC6 but stimulated the interaction between CIDEC and PCAF in the 3T3-L1 adipocytes. Data represent results from at least 3 independent experiments. (I) OA induced PCAF and CIDEC interaction in a dose-dependent manner. Data represent results from at least 3 independent experiments. (J) OA inhibited CIDEC and HDAC6 interaction in a dose-dependent manner. Data represent results from at least 3 independent experiments. (K) OA induced PCAF and CIDEC interaction but inhibited HDAC6 and CIDEC interaction. Data represent results from at least 3 independent experiments. (L) PCAF showed high binding affinity to the acetylation-defective form of CIDEC. Data represent results from at least 3 independent experiments. (M) HDAC6 had high affinity to the acetylation-mimicking form CIDEC. Data represent results from at least 3 independent experiments. 
GWAT of HFD-fed mice, obese mice, monkeys, and humans. HDAC6 and HDAC10 protein levels were also markedly reduced in the BAT of obese and HFD-fed mice. Interestingly, Hdac6 and Hdac1O mRNA levels were only moderately decreased in the GWAT of the obese mice. As the stability of HDAC6 was not controlled by the ubiquitin-dependent protein degradation pathway (data not shown), the significant reduction in HDAC6 protein levels in the adipose tissue of the $o b / o b$ or HFD-fed mice may be due to more complex regulatory mechanisms, such as translational regulation or specific protein degradation pathways activated in response to long-term FA exposure and/or increased lipid storage in the adipocytes.

Consistent with a negative role of HDAC6 in lipid storage, animals with adipose tissue-specific knockout of Hdac6 showed enlarged LDs and increased fat accumulation in the GWAT and BAT. Hdac6 deficiency resulted in slightly lower lipolysis but did not affect other lipid metabolic pathways, including FA uptake, FA biosynthesis, and TAG synthesis. The increased fat accumulation may be due to the lower metabolic activity in the adipose-specific Hdac6-deficient animals, as these animals displayed reduced oxygen consumption, lower respiration exchange, and lower energy expenditure. The lower metabolic activity may be partially contributed by reduced mitochondrial activity due to the lower expression of genes in the mitochondrial oxidative pathway in the BAT and GWAT of animals with adipose-specific Hdac6 deficiency. As we did not observe differences in food intake and physical activity between control and Hdac6 AKO mice, it was not likely that the adipose-specific knockout of Hdac6 influenced the CNS activity.

The GTT and ITT experiments showed that animals with an adipose-specific Hdac6 deficiency exhibited decreased insulin sensitivity. The hyperinsulinemic-euglycemic clamp experiment further confirmed the insulin-resistant phenotype of these animals, as they had decreased glucose infusion, glucose disposal, and insulin-stimulated GDRs. Interestingly, these animals did not show changes in HGP or circulating FFA levels. Therefore, Hdac6 deletion in the adipose tissue led to systemic and skeletal muscle insulin resistance. The reduced secretion of adiponectin and increased expression of inflammatory cytokines in the Hdac6 AKO animals may contribute to their reduced systemic insulin sensitivity.

We found that CIDEC, a protein responsible for lipid storage and LD fusion in adipocytes, is a downstream target of HDAC6 based on the following evidence. First, levels of acetylated CIDEC were increased in the adipose tissue of Hdac6-knockout mice. Knockdown of HDAC6 in other cell types also reduced levels of ectopically expressed CIDEC acetylation. We also identified PCAF as a specific acetyltransferase for CIDEC and K56 as the specific acetylation site. HDAC6 can antagonize the effect of PCAF-induced CIDEC acetylation. Importantly, CIDEC acetylation enhances its stability by inhibiting its ubiquitination and degradation. Functionally, LD fusion and growth and lipid storage activities were increased with expression of the acetylation-mimicking CIDEC mutant and in Hdac6-depleted adipocytes.

Interestingly, we found that FA can effectively enhance CIDEC stability by increasing its acetylation. FA-induced CIDEC acetylation was mediated by PCAF and inhibited by HDAC6. Notably, CIDEA, a close homolog of CIDEC, was also acetylated in response to FA treatment. FA-induced acetylation may repre- sent a general mechanism for the regulation of lipid metabolism in response to dietary FAs. Mechanistically, we have obtained clear evidence showing that FA-induced CIDEC acetylation was regulated by its ability to modulate the dynamic "ping-pong" association between CIDEC and PCAF and CIDEC and HDAC6. Under FA-free conditions, HDAC6 exhibited a stronger association with CIDEC, and this association decreased with the addition of FAs. By contrast, the association between PCAF and CIDEC increased in the presence of FAs in a dose-dependent manner. Imaging and biochemical fractionation analyses showed that PCAF and CIDEC were colocalized in the ER. By contrast, HDAC6 was only detected in the ER fractions when it was coexpressed with CIDEC, and HDAC6 was dissociated from the ER in the presence of FAs.

How do FAs regulate the interactions between CIDEC and HDAC6 and CIDEC and PCAF? In adipocytes, FAs are delivered to ER and then converted into phospholipids, TAG, or other intermediate metabolites through various enzymes in the lipid metabolic pathway. We showed that the ER is the primary site for FA-induced CIDEC acetylation. Importantly, we observed that the depletion of enzymes involved in TAG synthesis, such as a lipin 1 deficiency, results in reduced CIDEC acetylation. Therefore, TAG synthesis is required for this regulatory cascade. TAG may be able to directly disrupt the interaction between CIDEC and HDAC6 or promote the CIDEC-PCAF association. Alternatively, newly synthesized TAG may form a subdomain in the ER and promote nascent LD formation, resulting in changes in CIDEC configuration and dissociation of the CIDEC-HDAC6 interaction. In addition, ER- but not LD-associated CIDEC was ubiquitinated in vivo, suggesting that the configuration of CIDEC was different in these two subcellular organelles. Because PCAF binds to acetylated CIDEC at lower levels, acetylated CIDEC will be released from the ER and mobilized to the LD surface, where it promotes LD fusion and lipid storage.

Although the relative stoichiometry of K56 acetylation in CIDEC was not very high in control mouse adipocytes, it was dramatically increased in Hdac6-depleted or FA-treated adipocytes, consistent with the role of HDAC6 in mediating FA-induced CIDEC acetylation. The low stoichiometry of acetylated CIDEC is probably due to the limited availability of acetyl-CoA, as the primary site of CIDEC acetylation is on the ER. This is also consistent with the previously observed relatively low stoichiometry of acetylation of many proteins, in particular cytosolic proteins (36-38). Intriguingly, we observed that acetylated CIDEC is highly enriched at the LD fraction (Figure 7F). This local enrichment of acetylated CIDEC may dramatically increase the stoichiometry of CIDEC acetylation. In addition, acetylation of CIDEC at K56 may lead to its conformational change and render it inaccessible for ubiquitination and degradation. Therefore, we postulate that this local enrichment of acetylated CIDEC on LDs could dramatically enhance its stoichiometry and induce a conformational change to prevent it from undergoing ubiqutin-mediated protein degradation. In addition, another acetylation site (K34) on CIDEC may cooperate with K56 acetylation to control its stability and enhance its function in promoting LD fusion and lipid storage.

Previous research has shown that a Cidec deficiency results in reduced LD fusion activity, reduced lipid storage, and increased lipolysis in GWAT. When crossed to leptin-deficient $o b / o b$ or BATless obese mice, Cidec-deficient mice displayed hepatic steatosis 

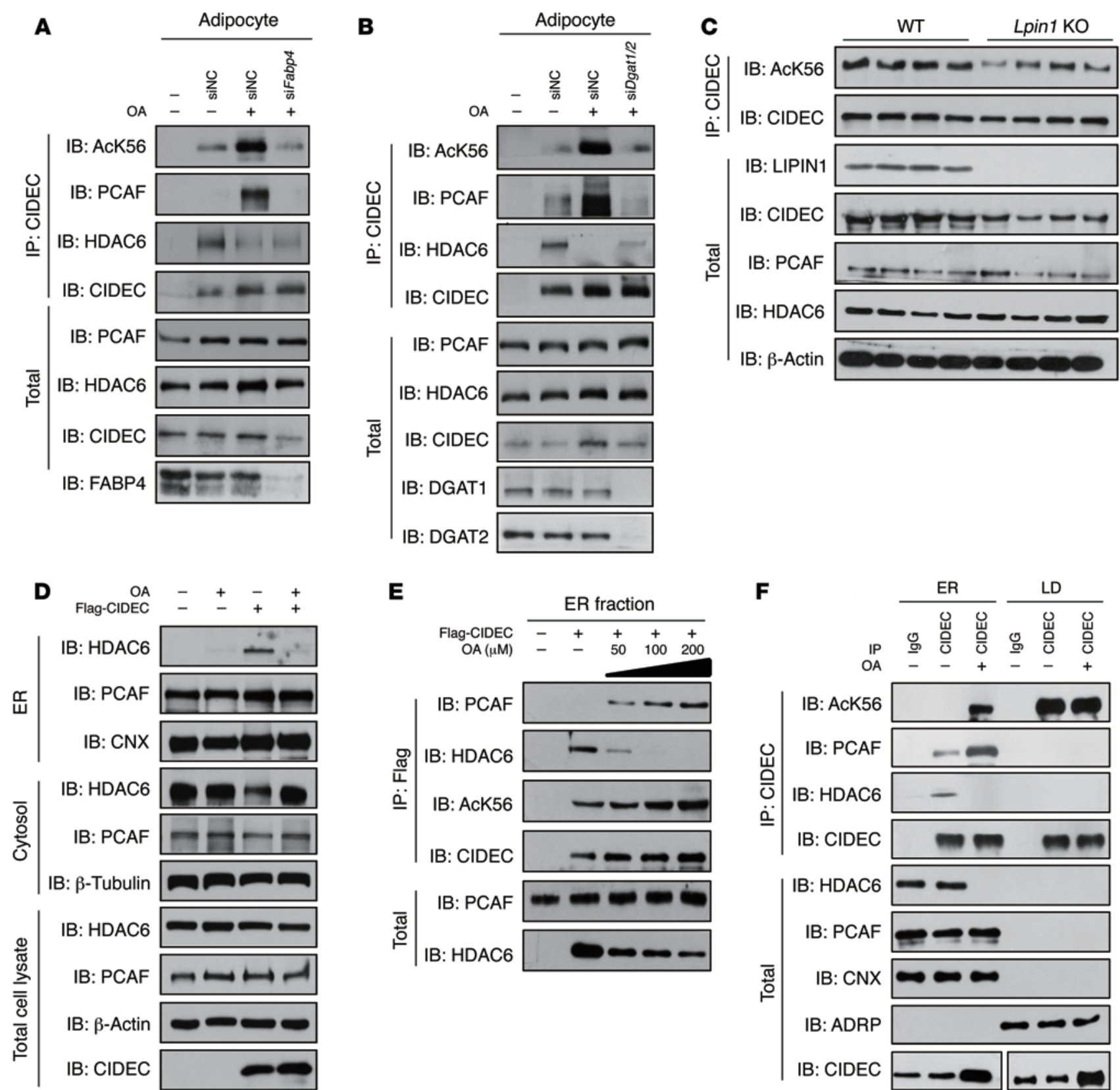

$\mathbf{F}$
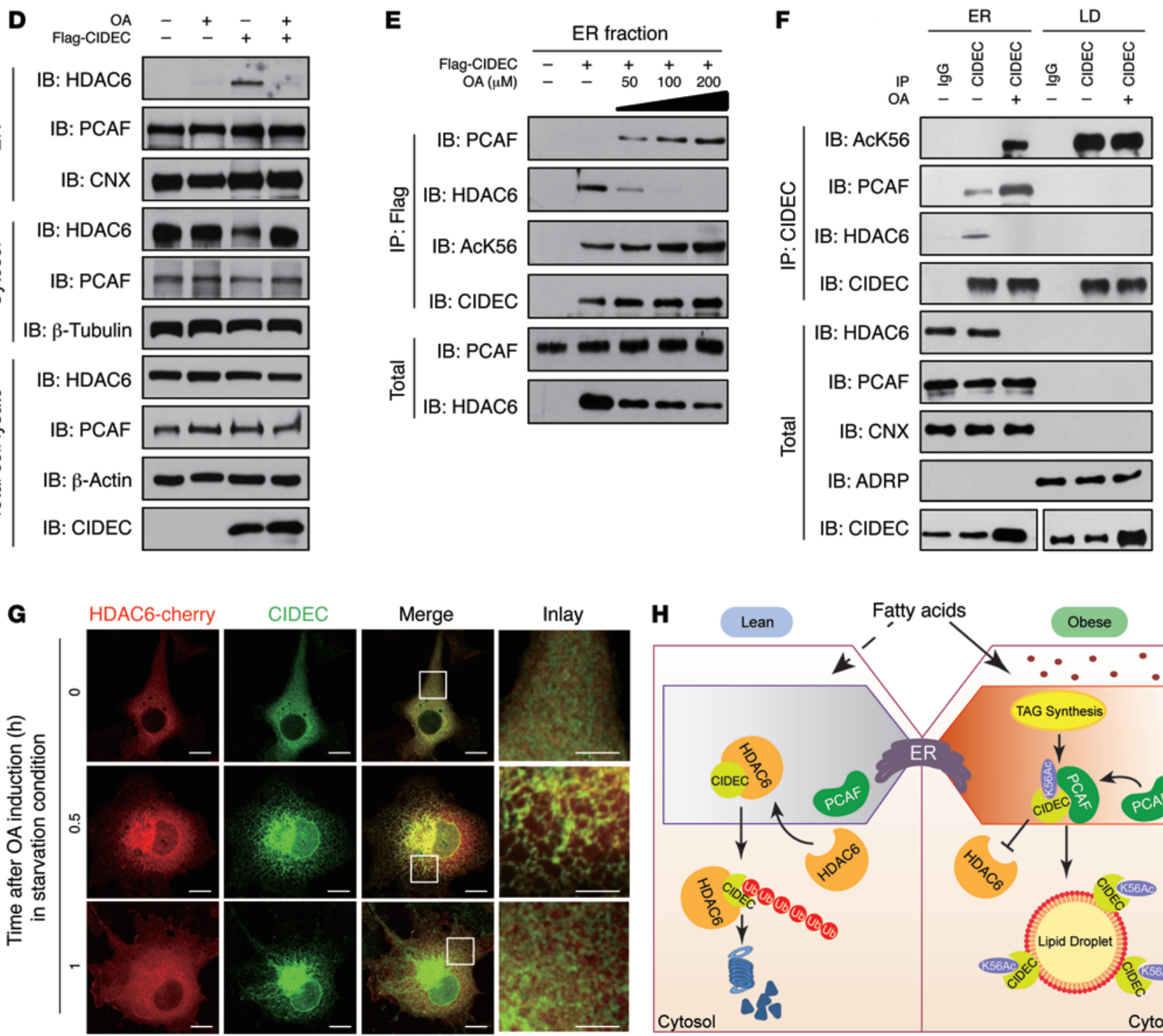
Figure 7. FA conversion into TAG in the ER is vital for it to induce CIDEC acetylation. (A) FA-induced CIDEC acetylation and the dynamic interaction among CIDEC, PCAF, and HDAC6 were disrupted by the depletion of Fabp4. Data represent results from 3 independent experiments. (B) FA-induced CIDEC acetylation and HDAC6, PCAF dynamic interaction with CIDEC was depended on DGAT activity. Data represent results from 3 independent experiments. (C) Levels of CIDEC protein and its acetylation were decreased in the GWAT of lipin 1-deficient mice ( $L$ pin1 KO) $(n=4$ mice per group). (D) HDAC6 is recruited to the ER in the presence of CIDEC and dissociated from ER in the presence of OA. Data represent results from at least 3 independent experiments. (E) The ER is the site of subcellular localization of FA-induced dynamic association among CIDEC, PCAF, and HDAC6. Data represent results from at least 3 independent experiments. (F) The ER is the site for OA-induced CIDEC acetylation and the dynamic interaction among CIDEC, PCAF, and HDAC6 in 3T3-L1 adipocytes. Data represent results from at least 3 independent experiments. (G) Representative images showing the colocalization of HDAC6 (red) and CIDEC (green) in the presence of OA. Scale bars: $5 \mu \mathrm{m}$ and $2 \mu \mathrm{m}$ (insets). Data represent results from at least 5 independent experiments. (H) Model depicting the role of HDAC6 in mediating FA-induced CIDEC acetylation and obesity development in adipocytes. When extracellular levels of FAs are low, CIDEC is associated with HDAC6, resulting in its lower acetylation and rapid degradation. In the presence of high levels of FAs, HDAC6 is dissociated from CIDEC, resulting in the association between CIDEC and PCAF and increased CIDEC acetylation. Acetylated CIDEC is mobilized to LD to promote fusion and lipid storage.

and insulin resistance (33). Interestingly, CIDEA, CIDEC, and PLIN1 expression is positively correlated with insulin sensitivity in humans. Therefore, the lipid storage capacity of adipose tissues must be carefully monitored. The moderate lipid storage observed when CIDEC is expressed at normal or slightly higher levels is beneficial for the animal. Excess lipid storage in adipose tissue (under obesity conditions when CIDEC is highly expressed) or very low lipid storage (CIDEC-deficient conditions) leads to insulin resistance. Although we cannot rule out the involvement of other downstream targets of HDAC6, CIDEC at least in part contributes to its role in controlling lipid storage and obesity development.

Based on the above evidence, we propose a model to explain the molecular mechanism of FA-induced CIDEC acetylation and obesity development (Figure 7H). In the absence of extracellular FAs, CIDEC is associated with HDAC6 in the ER, resulting in lower levels of CIDEC acetylation and its rapid degradation. Consequently, LDs are smaller, and lipid storage is reduced in adipocytes. In the presence of increased extracellular FA levels, FAs are transported into the ER and converted into TAG, resulting in the dissociation of CIDEC and HDAC6 and the association of CIDEC and PCAF. Acetylated CIDEC is released from the ER and transported to LDs, where it promotes LD fusion and growth and lipid storage. Therefore, protein acetylation serves as a convergence point for the cellular responses to dietary FAs and the development of obesity. Our current findings also suggest that pharmaceutical intervention with HDAC6 might be a promising therapeutic approach to combat obesity and its related diseases.

\section{Methods}

Details on plasmid construction, antibodies, reagents, recombinant protein, cell culture, RNAi, transfection, immunofluorescence staining, live cell imaging, immunoprecipitation, and MS; and lists of primers and siRNAs are described in Supplemental Methods.
MS. Samples for proteomics analysis were prepared as previously described (34). For LC-MS/MS analysis, digestion products were separated by a 60 -minute gradient elution at a $0.250-\mu \mathrm{l} / \mathrm{min}$ flow rate with a Thermo-Dionex Ultimate 3000 HPLC system, which was directly interfaced with a Thermo LTQ Orbitrap Velos mass spectrometer. The analytical column was a homemade fused silica capillary column (75 $\mu \mathrm{m}$ ID, $150 \mathrm{~mm}$ length; Upchurch) packed with C-18 resin (300 ̊, $5 \mu \mathrm{m}$; Varian). Mobile phase A consisted of $0.1 \%$ formic acid, and mobile phase B consisted of $100 \%$ acetonitrile and $0.1 \%$ formic acid. An LTQ Orbitrap mass spectrometer was operated in the data-dependent acquisition mode using Xcalibur 2.0.7 software. A single full-scan mass spectrum (400-1,800 m/z, 30,000 resolution) was acquired, followed by 20 data-dependent MS/MS scans in an ion trap at $35 \%$ normalized collision energy.

In vitro acetyltransferase assays. CIDEC-MBP recombinant protein was subjected to immunoprecipitation with MBP beads. The immunoprecipitates were mixed with a final volume $50 \mu$ of acetylation buffer (40 mM Tris- $\mathrm{HCl}$ [pH 8.0], 0.1 M NaCl, $0.1 \mathrm{mM}$ EDTA, $1 \mathrm{mM}$ phenylmethylsulfonyl fluoride, $1 \mu \mathrm{M}$ TSA, $0.1 \mathrm{mM}$ acetyl-CoA, $2 \mathrm{mM}$ sodium butyrate, and $2 \mathrm{mM}$ DTT) containing $1 \mu \mathrm{g}$ of recombinant $\mathrm{PCAF}_{352-832}{ }^{-}$ GST and were incubated for 60 minutes at $30^{\circ} \mathrm{C}$. The reaction was terminated by the addition of $40 \mu \mathrm{l}$ of $2 \times$ SDS sample buffer, the samples were boiled, and portions $(10 \mu \mathrm{l})$ of each mixture were subjected to immunoblot analysis.

Isolation of ER and LD fractions. ER was isolated from 293T cells treated with OA or 3T3-L1 adipocytes differentiated for 8 days according to the procedures for the Endoplasmic Reticulum Isolation Kit (Sigma-Aldrich). In brief, cells were detached and washed with PBS; and hypotonic extraction buffer (10 mM HEPES, pH 7.8, 1 mM EGTA, 25 $\mathrm{mM} \mathrm{KCl}$ ) was used to allow the cells to suspend and swell before they were centrifuged at $600 \mathrm{~g}$ for 5 minutes. The pellet was suspended in isotonic extraction buffer (10 mM HEPES, pH 7.8, $250 \mathrm{mM}$ sucrose, $1 \mathrm{mM}$ EGTA, $25 \mathrm{mM} \mathrm{KCl}$ ) and homogenized in a 7-ml Dounce homogenizer with 10 strokes. The homogenate was centrifuged at 1,000 $\mathrm{g}$ for 10 minutes, and the supernatant was then centrifuged at $12,000 \mathrm{~g}$ for 15 minutes. After the lipid pad was discarded, the supernatant was the post-mitochondrial fraction. The crude ER was the pellet collected after centrifugation of the post-mitochondrial fraction at $100,000 \mathrm{~g}$ for 60 minutes. All procedures were performed at $4^{\circ} \mathrm{C}$, and protease inhibitors cocktails (Roche) were added.

The LD fraction was isolated according to a previously described method (39), with slight modifications. In brief, 8-day-differentiated 3T3-L1 adipocytes or 293T cells treated with OA were harvested, washed with PBS, and resuspended in TES buffer (20 mM Tris, pH 7.4, $1 \mathrm{mM}$ EDTA, and $250 \mathrm{mM}$ sucrose). Cells were homogenized with a Dounce homogenizer with a loose-fitting pestle 25 times. The postnuclear supernatant after centrifugation at $1,000 \mathrm{~g}$ was overlaid with floating buffer $\left(100 \mathrm{mM} \mathrm{NaCl}, 20 \mathrm{mM}\right.$ Tris, $\mathrm{pH} 7.4$, and $\left.1.5 \mathrm{mM} \mathrm{MgCl}_{2}\right)$ and centrifuged at 200,000 $\mathrm{g}$ for 1 hour. The pellet and middle gradient were collected as total membrane and cytosol, respectively. The LDs on the top were collected and re-floated using the 2-layer gradient mentioned above for another 30 minutes at 200,000 $\mathrm{g}$. All procedures were performed at $4^{\circ} \mathrm{C}$, and protease inhibitor cocktails (Roche) were added. Protein concentration was determined using the Bradfold method (Bio-Rad).

TAG measurement. The measurement method for TAG in adipose tissue and liver was the same as described previously (30). For quantitative analysis, adipose and hepatic TAG spots of samples and 
standard on TLC plates (Sigma-Aldrich) were scanned and quantified using Quantity One software (Bio-Rad). TAG levels were normalized with the protein concentration of each sample.

Generation of adipose tissue-specific knockout mice and animal maintenance. The Hdac6 adipose tissue-specific knockout mice were generated on a C57BL/6J background. We generated a conditional Hdac6 allele $\left(H d a c 6^{f / f l}\right)$ by engineering loxP sites flanking exons 9 and 14 . We next

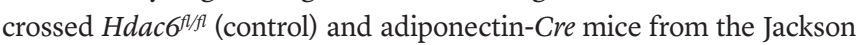
Laboratory to generate mice with adipocyte-specific knockout of Hdac6 (AKO). Genotyping primer sequences used to confirm $H d a c 6^{\text {fl/l }}$ were 5'-CATGGGTTTGGCAGGAGGCAA-3' and 5'-TGTGGGATCTCCTGAACTGG-3'. The primer sequences used to test adiponectin-Cre were 5'-CATGGGTTTGGCAGGAGGCAA-3' and GAACGCTAGAGCCTGTTTTGCACGTTC. Routine maintenance of mouse strains was performed in the laboratory animal facility at Tsinghua University. All of the mice used in the studies were male. The animals were fed ND (5053, PicoLab Rodent Diet 20, Research Diets) and HFD (58\% of kilocalories from fat; D12331, Research Diets) for 8 weeks.

Statistics. Data were subjected to statistical analysis with GraphPad Prism 5 (GraphPad Software) and were plotted by AI Illustrator (Adobe). Results represent the mean \pm SEM of at least 3 independent experiments as indicated in the figure legends. Two-tailed Student's $t$ test based on ANOVA was used for 2-group comparisons. For multiple comparisons made in Figure 1, F and G, and Figure 5, B, C, F, and $\mathrm{G}$, we conducted 1-way ANOVA with Dunnett's correction to analyze differences among the control group and 1 or more independent treatment groups. Differences were considered statistically significant at $P<0.05$, with significance indicated in figures as ${ }^{*} P<0.05,{ }^{*} P<$ $0.01,{ }^{* *} P<0.001$. NS represents no significant difference. We used 3 different statistical methods for comparison of oxygen consumption, including a direct comparison method, a ratio-based method, and ANCOVA using a multiple linear regression model. Statistical procedures in the 3 methods are discussed in detail in Supplemental materials. We used 2-way repeated-measures ANOVA to evaluate the data in Figure 1E (left), Figure 4A, and Supplemental Figure 4A with HolmŚídák post hoc test $\left({ }^{\#} P<0.05,{ }^{\# \#} P<0.01\right.$, and ${ }^{\# \#} P<0.001$ in these figures indicate that the 2 groups have significant difference).

Study approval. The Center of Biomedical Analysis at Tsinghua University was accredited by the Association for Assessment and
Accreditation of Laboratory Animal Care International (AAALAC) and the IACUC. All animal protocols were approved by the animal facility at Tsinghua University. The human study was approved by Air Force General Hospital, PLA, and Shanghai Sixth People's Hospital. Human patients who provided fat tissues were informed and consented with this study. The rhesus monkey study was approved by the Institute of Molecular Medicine, Peking University.

\section{Author contributions}

HQ and YC designed and performed most of the experiments, analyzed the data, and wrote the manuscript. FJC and LY helped with the image analysis. HD and YG helped with MS analysis. WX helped with protein purification. PL and SH helped with insulin clamp experiments. J. Li helped to generate Lpin1 $^{-1-}$ mice. J. Liu performed the experiments. HY, XZ, Hongchao Zhang, Haihong Zhang, YB, RPX, and WJ helped to collect human and monkey fat samples. LZ, LS, ZN, JY, and LY participated in the experimental design, discussion of results, and manuscript preparation. SZ helped with plasmid and reagent preparation. PL was responsible for forming the hypothesis, project development, data coordination, and writing, finalizing, and submitting the manuscript. All authors discussed and approved the manuscript.

\section{Acknowledgments}

We thank Xianming Pan of Tsinghua University for help with statistical analysis. We are grateful to members of the PL laboratory at Tsinghua University and Shengcai Lin for critical reading of the manuscript. This work was supported by grants from the National Natural Science Foundation of China (grant 31430040); National Basic Research Program (2013CB530602 and 2016YFA0502002); China Postdoctoral Science Foundation (grant 2015M581079); and Foundation for Innovative Research Groups of the National Natural Science Foundation of China (grant 31621063); and the National Natural Science Foundation of China (grants 31690103 and 31501089).

Address correspondence to: Peng Li, School of Life Sciences, Tsinghua University, HaiDian District, Beijing 100084, China. Phone: 86.10.62797121; E-mail: li-peng@mail.tsinghua.edu.cn.
1. Guan KL, Xiong Y. Regulation of intermediary metabolism by protein acetylation. Trends Biochem Sci. 2011;36(2):108-116.

2. Chuderland D, Konson A, Seger R. Identification and characterization of a general nuclear translocation signal in signaling proteins. Mol Cell. 2008;31(6):850-861.

3. Xu W, Li Y, Liu C, Zhao S. Protein lysine acetylation guards metabolic homeostasis to fight against cancer. Oncogene. 2014;33(18):2279-2285.

4. Brooks CL, Gu W. Ubiquitination, phosphorylation and acetylation: the molecular basis for $\mathrm{p} 53$ regulation. Curr Opin Cell Biol. 2003;15(2):164-171.

5. Guarente L. The logic linking protein acetylation and metabolism. Cell Metab. 2011;14(2):151-153.

6. Iyer A, Fairlie DP, Brown L. Lysine acetylation in obesity, diabetes and metabolic disease. Immunol Cell Biol. 2012;90(1):39-46.

7. Winkler R, et al. Histone deacetylase 6 (HDAC6) is an essential modifier of glucocorticoid-induced hepatic gluconeogenesis. Diabetes. 2012;61(2):513-523

8. Mihaylova MM, Shaw RJ. Metabolic reprogramming by class I and II histone deacetylases. Trends Endocrinol Metab. 2013;24(1):48-57.

9. Zhang M, et al. HDAC6 deacetylates and ubiquitinates MSH2 to maintain proper levels of MutS $\alpha$. Mol Cell. 2014;55(1):31-46.

10. Boyault C, et al. HDAC6-p97/VCP controlled polyubiquitin chain turnover. EMBO J. 2006;25(14):3357-3366.

11. Seigneurin-Berny D, et al. Identification of components of the murine histone deacetylase 6 complex: link between acetylation and ubiquitination signaling pathways. Mol Cell Biol. 2001;21(23):8035-8044.

12. Kanno K, et al. Overexpression of histone deacetylase 6 contributes to accelerated migra- tion and invasion activity of hepatocellular carcinoma cells. Oncol Rep. 2012;28(3):867-873.

13. Oehme I, et al. Histone deacetylase 10 promotes autophagy-mediated cell survival. Proc Natl Acad Sci U S A. 2013;110(28):E2592-E2601.

14. Nagy Z, Tora L. Distinct GCN5/PCAF-containing complexes function as co-activators and are involved in transcription factor and global histone acetylation. Oncogene. 2007;26(37):5341-5357.

15. Jiang W, et al. Acetylation regulates gluconeogenesis by promoting PEPCK1 degradation via recruiting the UBR5 ubiquitin ligase. Mol Cell. 2011;43(1):33-44.

16. Lv L, et al. Acetylation targets the M2 isoform of pyruvate kinase for degradation through chaperone-mediated autophagy and promotes tumor growth. Mol Cell. 2011;42(6):719-730.

17. Zhang T, et al. Acetylation negatively regulates glycogen phosphorylase by recruiting protein 
phosphatase 1. Cell Metab. 2012;15(1):75-87.

18. von Meyenn F, et al. Glucagon-induced acetylation of Foxa2 regulates hepatic lipid metabolism. Cell Metab. 2013;17(3):436-447.

19. Park J, Morley TS, Kim M, Clegg DJ, Scherer PE. Obesity and cancer - mechanisms underlying tumour progression and recurrence. Nat Rev Endocrinol. 2014;10(8):455-465.

20. Krahmer N, Farese RV Jr, Walther TC. Balancing the fat: lipid droplets and human disease. $Е M B O$ Mol Med. 2013;5(7):905-915

21. Ducharme NA, Bickel PE. Lipid droplets in lipogenesis and lipolysis. Endocrinology. 2008;149(3):942-949.

22. Rosen ED, Spiegelman BM. What we talk about when we talk about fat. Cell. 2014;156(1-2):20-44.

23. Walther TC, Farese RV. Lipid droplets and cellular lipid metabolism. Annu Rev Biochem. 2012;81:687-714.

24. Wilfling F, et al. Triacylglycerol synthesis enzymes mediate lipid droplet growth by relocalizing from the ER to lipid droplets. Dev Cell. 2013;24(4):384-399.

25. Xu N, et al. The FATP1-DGAT2 complex facilitates lipid droplet expansion at the ER-lipid drop- let interface. JCell Biol. 2012;198(5):895-911.

26. Sun Z, et al. Perilipin1 promotes unilocular lipid droplet formation through the activation of Fsp27 in adipocytes. Nat Commun. 2013;4:1594.

27. Wu L, et al. Rab8a-AS160-MSS4 regulatory circuit controls lipid droplet fusion and growth. Dev Cell. 2014;30(4):378-393.

28. Puri V, et al. Fat-specific protein 27 , a novel lipid droplet protein that enhances triglyceride storage. J Biol Chem. 2007;282(47):34213-34218.

29. Gong J, et al. Fsp27 promotes lipid droplet growth by lipid exchange and transfer at lipid droplet contact sites. J Cell Biol. 2011;195(6):953-963.

30. Toh SY, et al. Up-regulation of mitochondrial activity and acquirement of brown adipose tissue-like property in the white adipose tissue of fsp27 deficient mice. PLoS One. 2008;3(8):e2890.

31. Nishino N, et al. FSP27 contributes to efficient energy storage in murine white adipocytes by promoting the formation of unilocular lipid droplets. JClin Invest. 2008;118(8):2808-2821.

32. Puri V, Czech MP. Lipid droplets: FSP27 knockout enhances their sizzle. JClin Invest. 2008;118(8):2693-2696.

33. Zhou L, et al. Insulin resistance and white adi- pose tissue inflammation are uncoupled in energetically challenged Fsp27-deficient mice. Nat Commun. 2015;6:5949.

34. Zhao S, et al. Regulation of cellular metabolism by protein lysine acetylation. Science. 2010;327(5968):1000-1004.

35. Rardin MJ, et al. Label-free quantitative proteomics of the lysine acetylome in mitochondria identifies substrates of SIRT3 in metabolic pathways. Proc Natl Acad Sci U S A. 2013;110(16):6601-6606.

36. Weinert BT, et al. Acetylation dynamics and stoichiometry in Saccharomyces cerevisiae. Mol Syst Biol. 2015;11(10):833.

37. Baeza J, et al. Stoichiometry of site-specific lysine acetylation in an entire proteome. J Biol Chem. 2014;289(31):21326-21338.

38. Weinert BT, Moustafa T, Iesmantavicius V, Zechner R, Choudhary C. Analysis of acetylation stoichiometry suggests that SIRT3 repairs nonenzymatic acetylation lesions. $E M B O J$. 2015;34(21):2620-2632.

39. Bartz R, et al. Dynamic activity of lipid droplets: protein phosphorylation and GTPmediated protein translocation. JProteome Res. 2007;6(8):3256-3265. 